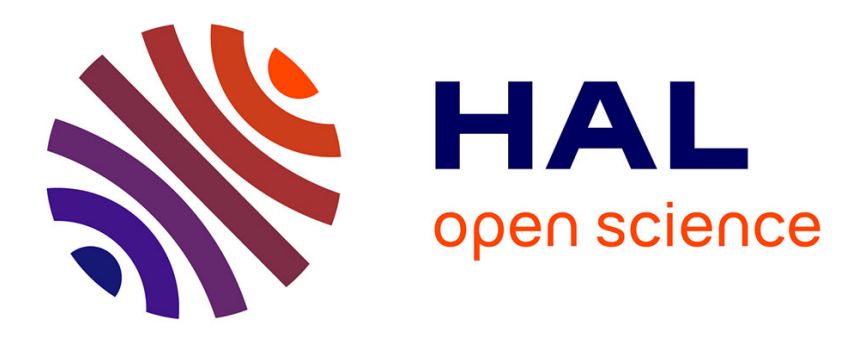

\title{
Stopping Behaviors of Naïve and Non-Committed Sophisticated Agents when They Distort Probability
}

Yu-Jui Huang, Adrien Nguyen-Huu, Xun Yu Zhou

\section{To cite this version:}

Yu-Jui Huang, Adrien Nguyen-Huu, Xun Yu Zhou. Stopping Behaviors of Naïve and Non-Committed Sophisticated Agents when They Distort Probability. 2017. hal-01586655

\section{HAL Id: hal-01586655 \\ https://hal.science/hal-01586655}

Preprint submitted on 13 Sep 2017

HAL is a multi-disciplinary open access archive for the deposit and dissemination of scientific research documents, whether they are published or not. The documents may come from teaching and research institutions in France or abroad, or from public or private research centers.
L'archive ouverte pluridisciplinaire HAL, est destinée au dépôt et à la diffusion de documents scientifiques de niveau recherche, publiés ou non, émanant des établissements d'enseignement et de recherche français ou étrangers, des laboratoires publics ou privés. 


\title{
Stopping Behaviors of Naïve and Non-Committed Sophisticated Agents when They Distort Probability*
}

\author{
Yu-Jui Huang ${ }^{\dagger} \quad$ Adrien Nguyen-Huu ${ }^{\ddagger} \quad$ Xun Yu Zhou ${ }^{\S}$ \\ September 13, 2017
}

\begin{abstract}
We consider the problem of stopping a diffusion process with a payoff functional involving probability distortion. The problem is inherently time-inconsistent as the level of distortion of a same event changes over time. We study stopping decisions of naïve agents who reoptimize continuously in time, as well as equilibrium strategies of sophisticated agents who anticipate but lack control over their future selves' behaviors. When the state process is one dimensional and the payoff functional satisfies some regularity conditions, we prove that any equilibrium can be obtained as a fixed point of an operator. This operator represents strategic reasoning that takes the future selves' behaviors into account. In particular, we show how such strategic reasoning may turn a naïve agent into a sophisticated one. Finally, when the diffusion process is a geometric Brownian motion we derive stopping strategies of these two types of agent for various parameter specifications of the problem, illustrating rich behaviors beyond the extreme ones such as "never-stopping" or "never-starting".
\end{abstract}

JEL: G11, I12

MSC (2010): 60G40, 91B06

Keywords: Optimal stopping, probability distortion, time inconsistency, naïve and sophisticated agents, equilibrium stopping law

${ }^{*}$ Y.-J. Huang gratefully acknowledges financial support from National Science Foundation (DMS-1715439) and a start-up grant at the University of Colorado (11003573). A. Nguyen-Huu has received support from the Energy \& Prosperity Chair and Risk Foundation. X. Y. Zhou gratefully acknowledges financial support through a start-up grant at Columbia University and through Oxford-Nie Financial Big Data Lab.

${ }^{\dagger}$ Department of Applied Mathematics, University of Colorado, Boulder, CO 80309, USA, yujui.huang@colorado.edu

${ }^{\ddagger}$ LAMETA, Univ. Montpellier, CNRS, INRA, Montpellier SupAgro, Univ. Paul Valéry, Montpellier, France, adrien.nguyen-huu@umontpellier.fr

${ }^{\S}$ Department of Industrial Engineering and Operations Research, Columbia University, New York, New York 10027, USA, xz2574@columbia.edu 


\section{Introduction}

Optimal stopping is to determine the best (random) time to stop a stochastic process so as to maximize a given payoff arising from the stopped state of the process. Applications of such a timing problem are abundant including stock trading (e.g. the best time to sell a stock), option pricing (e.g. American options) and real options (e.g. the best time to invest in a project). Two main classical approaches to solving optimal stopping problems are dynamic programming and martingale theory, which are both based foundationally on the assumption of time-consistency, namely, any optimal stopping rule planned today remains optimal tomorrow.

The assumption of time-consistency is rooted in the premise that an individual's preferences are consistent over time and will not change as time goes by or circumstances evolve. However, this premise is all too vulnerable to stand the test of reality. A gambler may have planned initially to leave the casino after having made $\$ 1000$, but then decides to go on gambling when this target has been reached. This is because the gambler's risk preference has changed after winning $\$ 1000$; he has become more risk-seeking as the result of the triumph. Indeed, extensive empirical and experimental studies all point to the fact that time-inconsistency is the rule rather than the exception.

In the absence of time-consistency, whatever an "optimal" plan derived at this moment is generally not optimal evaluated at the next moment; hence there is no such notion of a "dynamically optimal strategy" good for the entire planning horizon as is the case with a time-consistent model. Economists, starting from Strotz (1955-56), instead have described the responses or behaviors of three types of individuals when facing a time-inconsistent situation. Type 1, the naïve agent, chooses whatever seems to be optimal with respect to his current preference, without knowing the fact that his preferences may change over time. Type 2 is the pre-committed agent, who optimizes only once at the initial time and then sticks to the resulting plan in the future. Type 3, the sophisticated agent, is aware of the fact that his "future selves" will overturn his current plan (due to the lack of commitment) and selects the best present action taking the future disobedience as a constraint. The resulting strategy is called a (subgame perfect) equilibrium from which no incarnations of the agent have incentive to deviate.

Thus, a time-inconsistent model is descriptive as opposed to its time-consistent counterpart that is mainly prescriptive. The objective of the former is to describe behaviors of the different types of agents, rather than to advise on the best course of action 1 .

In this paper we study the stopping problem when probability is distorted. Experimental evidence supports the phenomenon of probability distortion (or weighting); in particular that people inflate the small probabilities of occurrence of both extremely favourable and extremely unfavorable events. Behavioral theories such as rank-dependent utility (RDU) and cumulative prospect theory (CPT) include probability distortion as a key component and consider it as a part of the risk preference. On the other hand, the level of probability distortion associated with a same future event changes over time because the conditional probability of that event evolves dynamically and the distortion is nonlinear. Therefore time-inconsistency is inherent in the presence of (non-trivial) probability distortion.

\footnotetext{
${ }^{1}$ The strategies of the three types of agents coincide if the problem is time consistent.
} 
The aim of this paper is to study the stopping strategies of Type 1 (naïve) and Type 3 (sophisticated) agents. Pre-committed strategies (i.e. those of Type 2's) have been investigated and obtained thoroughly for geometric Brownian motions by $\mathrm{Xu}$ and Zhou (2013)2, which are conceptually and technically useful for the present paper.

A naïve stopping law is one such that, at any given time and state, it coincides with the optimal stopping law at that particular pair of time and state. Definition of equilibrium strategies in the continuous-time setting, on the other hand, is more subtle. Starting with Ekeland and Lazrak (2006), and followed by e.g. Ekeland and Lazrak (2006), Yong (2012), $\mathrm{Hu}$ et al. (2012), Björk et al. (2017), and $\mathrm{Hu}$ et al. (2017), an equilibrium for a control problem is defined as one that satisfies a first-order inequality condition on some spike variation of the equilibrium. Ebert et al. (2017) apply this definition to a stopping problem by turning the latter into a control problem. However, it remains a problem to rigorously establish the equivalence between this first-order condition and the zeroth-order condition in the original definition of a subgame perfect equilibrium.

In this paper we follow the formulation of Huang and Nguven-Huu (2016) to define an equilibrium stopping law (although therein a different stopping problem with a nonexponential discount factor featuring decreasing impatience is considered). The idea of this formulation is that, for any given stopping law, the sophisticated agent improves it by a level of strategic reasoning through anticipating his future selves' behaviors. The agent then performs additional levels of similar reasoning until he cannot further improve it, which is an equilibrium. Mathematically, an equilibrium is a fixed-point of an operator that represents one level of this strategic thinking.

The general existence of such a fixed-point is a largely open question. The first contribution of this paper is to prove that, assuming that the state follows a one-dimensional diffusion process and the payoff functional satisfies some (very mild) measurable and Fatou type conditions, any equilibrium strategy can be obtained from performing the aforementioned operator repeatedly on an initial stopping law and then taking the limit.

We then apply this result to the stopping problem of a geometric Brownian motion and a RDU type of payoff functional involving probability distortion, whose pre-committed stopping strategies have been studied thoroughly by $\mathrm{Xu}$ and Zhou (2013). Besides characterizing the stopping behaviors of the naïve and the sophisticated, we are particularly interested in the connection and potential transformation among the three type of agents: the naïve agent in effect solves a pre-committed problem at every state and time who, interestingly, may turn himself into a sophisticate if he carries out several levels (sometimes just one level) of the strategic reasoning. In this regard, the fixed-point characterization of the equilibrium strategies brings about a significant advantage over the first-order characterization, as the former describes the "reasoning process" with which an initial naïve strategy is changed into a sophisticated one.

Ebert and Strack (2015) show that under the assumptions leading to "skewness preference in the small", a naive following CPT will never stop "until the bitter end". These assumptions essentially ensure that probability distortion on small probabilities of big gains

\footnotetext{
${ }^{2}$ Due to time-inconsistency, dynamic programming or martingale approach does not work for deriving pre-committed strategies. Xu and Zhou (2013) develop a new approach with a combination of the so-called quantile/distribution formulation and Skorokhod's embedding theory to solve the problem.
} 
outweighs loss aversion; hence at any wealth level the agent always favors a small, rightskewed risk over the deterministic payoff resulting from the immediate stop. This is the reason why he ends up never stopping. The antithesis of this result is presented in Ebert and Strack (2017): assuming that there is an arbitrarily strong probability distortion on small probabilities of big losses and considering only two-threshold type of strategies 3 , a sophisticate will stop immediately or, equivalently, "never, ever getting started". This is because when the process is sufficiently close to the upper threshold the stopped state under the two-threshold strategy becomes highly left-skewed, which is unattractive to a CPT agent with a typical inverse-S shaped distortion function. Knowing that the future selves who are close to the upper threshold will not carry out this strategy, in equilibrium each current self will simply not start it.

The authors of these two papers acknowledge that both the "never stop" and "never start" behaviors are "extreme" representing "unrealistic predictions" under the CPT. In this paper, we complement their results by studying the cases in which the assumptions of Ebert and Strack (2015) and Ebert and Strack (2017) do not hold, and showing that the behaviors of the naives and sophisticates are far richer than the mere extreme ones. Indeed, depending on the parameters of the geometric Brownian motion and the payoff functional, both types of agents may want to start and end, relying on a (one) threshold-type stopping strategy. This suggests that the RDU model may offer more realistic prediction if we allow the distortion functions to take various shapes and, meanwhile, we take into consideration the intertwining relationship between the preference of the agent and the characteristics of the process he monitors.

The rest of the paper is organized as follows. In Section 2 we formulate a general timeinconsistent stopping problem, define the naïve and sophisticated equilibrium stopping laws, and introduce the operator that represents a strategic reasoning of a sophisticated agent. Section 3 characterizes the set of equilibrium stopping laws when the state process is one dimensional. In Section 4 we demonstrate how time-inconsistency may arise when the probability is distorted and the process is a geometric Brownian motion, followed by Section 5 in which we develop the theory for deriving sophisticated equilibrium strategies in the presence of time inconsistency. Section 6 is devoted to derivation of naïve and sophisticated strategies for a wide range of parameter specifications. Finally, Appendices collect technical proofs.

\section{Naïve and Equilibrium Stopping Laws}

This section sets out to introduce time-inconsistency for a general optimal stopping problem and the ways we deal with the issue. Although in the rest of this paper we focus on problems in which the probability is distorted, the discussion in this section is more general.

Consider a probability space $(\Omega, \mathcal{F}, \mathbb{P})$ that supports a stationary stochastic process $X$ : $\mathbb{R}_{+} \times \Omega \mapsto \mathbb{X} \subseteq \mathbb{R}^{d}$ with initial value $X_{0}=x \in \mathbb{X}$, where $\mathbb{X}$ is called the state space of $X$. We will constantly use the notation $X^{x}$ to emphasize the dependence of the process on the initial value $x$. We denote by $\mathcal{B}(\mathbb{X})$ the Borel $\sigma$-algebra of $\mathbb{X}$. Let $\mathbb{F}=\left\{\mathcal{F}_{t}\right\}_{t \geq 0}$ be the $\mathbb{P}$ -

\footnotetext{
${ }^{3}$ With a two-threshold type of strategy, the agent stops whenever the state process reaches $a$ or $b$ where $a<b$ are the two prescribed thresholds. Ebert and Strack (2017) consider pure Markovian strategies, which are essentially two-threshold strategies.
} 
augmentation of the natural filtration generated by $X$, and let $\mathcal{T}$ be the set of all $\mathbb{F}$-stopping times that may be infinite with positive probabilities. Note that $\mathcal{T}$ includes non-Markovian stopping times. 4 .

At any given state $x \in \mathbb{X}$, a decision-maker (an agent) needs to decide when to stop the process $X$. If he chooses to stop at $\tau \in \mathcal{T}$, he receives the payoff $J(x ; \tau)$, where $J: \mathbb{X} \times \mathcal{T} \mapsto \mathbb{R}$ is a given objective functional. If he decides to stop immediately, then he gets a payoff $u(x)$, where $u: \mathbb{X} \mapsto \mathbb{R}$ is some Borel measurable function. The agent intends to maximize his payoff by choosing an appropriate stopping time $\tau \in \mathcal{T}$, i.e., he aspires to achieve

$$
\sup _{\tau \in \mathcal{T}} J(x ; \tau)
$$

A well-studied example of the objective functional is the expected payoff

$$
J(x ; \tau):=\mathbb{E}\left[u\left(X_{\tau}^{x}\right)\right],
$$

whereas there can be many other forms.

As $\left\{X_{t}^{x}\right\}_{t \geq 0}$ evolves over time, one can reexamine and solve the optimal stopping problem (2.1) at every moment $t \geq 0$. A natural, conceptual question arises: suppose an optimal stopping time $\hat{\tau}_{x}$ can be found for each and every $x \in \mathbb{X}$, are $\hat{\tau}_{x}$ and $\hat{\tau}_{X_{t}^{x}}, t>0$, consistent with each other?

The notion of "consistency" in this question can be formalized as follows:

Definition 2.1 (Time-Consistency). Suppose an optimal stopping time $\hat{\tau}_{x} \in \mathcal{T}$ of the problem (2.1) exists for all $x \in \mathbb{X}$. We say problem (2.1) is time-consistent if for any $t>0$ and any $x \in \mathbb{X}$,

$$
\hat{\tau}_{x}=t+\hat{\tau}_{X_{t}^{x}} \quad \text { a.s. on }\left\{\hat{\tau}_{x}>t\right\}
$$

otherwise problem (2.1) is time-inconsistent.

Intuitively, time-inconsistency means that an optimal strategy planned at this moment may no longer be optimal at the next moment. It is well known that problem (2.1) with the expected payoff in (2.2) is time-consistent; hence one can apply classical approaches such as dynamic programming and martingale method to solve it; see e.g. Shirvaev (1978) and Karatzas and Shreve (1998). For a general objective functional, problem (2.1) is predominantly time-inconsistent - for example when probability distortion is involved or nonexponential discouting is applied.

Time-inconsistency renders the very notion of "dynamic optimization" null and void, for if one cannot commit his future selves to the optimal strategy he chooses today, then today's optimal strategy has little use in a dynamic context. More specifically, for any given state $x \in \mathbb{X}$ at $t=0$, suppose the agent finds an optimal stopping time $\hat{\tau}_{x}$. He actually wants, and indeed assumes, that all his future selves will follow $\hat{\tau}_{x}$, so that the optimal value $\sup _{\tau \in \mathcal{T}} J(x ; \tau)=J\left(x ; \hat{\tau}_{x}\right)$ can be attained. However, his future self at time $t>0$ would like

\footnotetext{
${ }^{4}$ Non-Markovian stopping is widely used in practice. For example, the "trailing stop" in stock trading is a non-Markovian selling rule. He et al. (2017) shows that in a casino gambling model under CPT pathdependent stopping strategies strictly dominate Markovian ones.

${ }^{5}$ In later sections we will focus on an objective functional when the probability is "distorted", as defined in (4.9) below.
} 
to follow his own optimal stopping time $\hat{\tau}_{X_{t}^{x}}$, which may not be consistent with $\hat{\tau}_{x}$ in the sense of (2.3). If the agent at time 0 does not have sufficient control over his future selves' behavior, $\hat{\tau}_{x}$ will not be carried out throughout, and the optimal value $\sup _{\tau \in \mathcal{T}} J(x ; \tau)$ as initially figured out at $t=0$ will thus not be attained.

As discussed in the introduction, three types of agents have been described in literature in the presence of time-inconsistency. A naïve agent simply follows an optimal stopping time $\hat{\tau}_{X_{t}^{x}}$ at every moment $t \geq 0$, without knowing the underlying time-inconsistency. A sophisticated agent who is aware of the time-inconsistency but lacks commitment, by contrast, works on consistent planning: he takes into account his future selves' behaviors, and tries to find a stopping strategy that, once being employed over time, none of his future selves has incentives to deviate from it. Finally, a sophisticated agent who is able to commit simply solves the problem once at $t=0$ and then sticks to the corresponding stopping plan. The problem of the last type, the so-called pre-committed agent, is actually a static (instead of dynamic) problem and has been solved in various contexts and, in the case of optimal stopping under probability distortion, by $\mathrm{Xu}$ and Zhou (2013). The goal of this paper is to study the behaviors of the first two types of agents. It turns out, as we will demonstrate, that the solutions of the two are interwound, and both depend heavily on that of the last type.

Now we provide precise formulations of the stopping strategies of the first two types of agents (hereafter referred respectively as the naïve and sophisticated agents). We first introduce the notion of stopping laws.

Definition 2.2 (Stopping Laws). A Borel measurable function $\tau: \mathbb{X} \mapsto\{0,1\}$ is called a (Markovian) stopping law. We denote by $\mathcal{T}(\mathbb{X})$ the collection of all stopping laws.

The notion of stopping laws is analogous to that of feedback control laws in control theory. A stopping law is independent of any state process; however for any given process, such a law induces a stopping decision (in response to any current state) in the following manner.

Given the process $X$ with $X_{0}=x$, each stopping law $\tau \in \mathcal{T}(\mathbb{X})$ governs when the agent stops $X$ : the agent stops at the first time when $\tau\left(X_{t}\right)$ yields the value 0 , i.e. at the moment

$$
\mathcal{L} \tau(x):=\inf \left\{t \geq 0: \tau\left(X_{t}^{x}\right)=0\right\}
$$

In other words, $\mathcal{L} \tau(x)$ is the stopping time induced by the stopping law $\tau$ when the current state of the process is $x$.

We first define the stopping law used by a naïve agent.

Definition 2.3 (Naïve Stopping Law). Denote by $\left\{\hat{\tau}_{x}\right\}_{x \in \mathbb{X}} \subset \mathcal{T}$ a collection of optimal stopping times of (2.1), while noting that $\hat{\tau}_{x}$ may not exist for some $x \in \mathbb{X}$. Define $\hat{\tau}: \mathbb{X} \mapsto$ $\{0,1\}$ by

$$
\hat{\tau}(x):= \begin{cases}0, & \text { if } \hat{\tau}_{x}=0, \\ 1, & \text { if } \hat{\tau}_{x}>0 \text { or } \hat{\tau}_{x} \text { does not exist. }\end{cases}
$$

If $\hat{\tau}$ is Borel measurable, we say that it is the naïve stopping law generated by $\left\{\hat{\tau}_{x}\right\}_{x \in \mathbb{X}}$. 
The stopping law $\hat{\tau} \in \mathcal{T}(\mathbb{X})$ defined above describes the behavior of a naïve agent. For any current state $x>0$, a naïve agent decides to stop or to continue simply by following the optimal stopping time $\hat{\tau}_{x}$, if such a stopping time exists. If $\hat{\tau}_{x}$ fails to exist at some $x \in \mathbb{X}$, we must have $\sup _{\tau \in \mathcal{T}} J(x ; \tau)>u(x)$ (otherwise, we can take $\hat{\tau}_{x}=0$ ). Although the optimal value $\sup _{\tau \in \mathcal{T}} J(x ; \tau)$ cannot be attained, the naïve agent can pick some $\tau \in \mathcal{T}$ with $\tau>0$ a.s. such that $J(x ; \tau)$ is arbitrarily close to $\sup _{\tau \in \mathcal{T}} J(x ; \tau)$, leading to $J(x ; \tau)>u(x)$. That is, a naïve agent determines that it is better to continue than to stop at $x \in \mathbb{X}$. This is why we set $\hat{\tau}(x)=1$ when $\hat{\tau}_{x}$ does not exist in the above definition 6 .

Remark 2.1. Time-consistency in Definition 2.1 can also be formulated as

$$
\hat{\tau}_{x}=\mathcal{L} \hat{\tau}(x) \quad \forall x \in \mathbb{X}
$$

which means that the moment at which a naïve agent will stop, i.e. $\mathcal{L} \hat{\tau}(x)$, is entirely the same as what the original pre-committed optimal stopping time (which was planned at time 0 when the process was in state $x), \hat{\tau}_{x}$, prescribes.

Now we turn to characterizing a sophisticated agent. Suppose he starts with an initial stopping law $\tau \in \mathcal{T}(\mathbb{X})$. At any current state $x \in \mathbb{X}$, the agent carries out the following game-theoretic reasoning: if all my future selves will follow $\tau \in \mathcal{T}(\mathbb{X})$, what is the best stopping strategy at current time in response to that? Since the agent at current time can only choose to stop or to continue, he simply needs to compare the payoffs resulting from these two different actions. If the agent stops at current time, he obtains the payoff $u(x)$ immediately. If the agent chooses to continue at current time, given that all his future selves will follow $\tau \in \mathcal{T}(\mathbb{X})$, the agent will eventually stop at the moment

$$
\mathcal{L}^{*} \tau(x):=\inf \left\{t>0: \tau\left(X_{t}^{x}\right)=0\right\},
$$

leading to the payoff

$$
J\left(x ; \mathcal{L}^{*} \tau(x)\right) .
$$

Note the subtle difference between the two stopping times, $\mathcal{L}^{*} \tau(x)$ and $\mathcal{L} \tau(x)$ : at any moment one may continue under the former even though the latter may instruct to stop.

Some conclusions can then be drawn: (i) The agent should stop at current time if $u(x)>$ $J\left(x ; \mathcal{L}^{*} \tau(x)\right)$, and continue at current time if $u(x)<J\left(x ; \mathcal{L}^{*} \tau(x)\right)$. (ii) For the case where $u(x)=J\left(x ; \mathcal{L}^{*} \tau(x)\right)$, the agent is indifferent between stopping and continuation at current time; there is therefore no incentive for the agent to deviate from the originally assigned stopping strategy $\tau(x)$ now. This is already the best stopping strategy (or law) at current time (subject to all the future selves following $\tau \in \mathcal{T}(\mathbb{X})$ ), which can be summarized as

$$
\Theta \tau(x):= \begin{cases}0, & \text { if } x \in S_{\tau}, \\ 1, & \text { if } x \in C_{\tau}, \\ \tau(x), & \text { if } x \in I_{\tau},\end{cases}
$$

\footnotetext{
${ }^{6}$ There is a technical question as to whether optimal stopping times $\left\{\hat{\tau}_{x}\right\}_{x \in \mathbb{X}}$ of (2.1) can be chosen such that $\hat{\tau}$ defined in (2.5) is Borel measurable. The answer is positive under the standard expected payoff formulation (2.2). For an objective functional under probability distortion, we will see in Sections 4 and 6 that $\hat{\tau}$ is measurable in all examples we explore.
} 
where

$$
\begin{aligned}
S_{\tau} & :=\left\{x \in \mathbb{X}: J\left(x ; \mathcal{L}^{*} \tau(x)\right)<u(x)\right\}, \\
C_{\tau} & :=\left\{x \in \mathbb{X}: J\left(x ; \mathcal{L}^{*} \tau(x)\right)>u(x)\right\}, \\
I_{\tau} & :=\left\{x \in \mathbb{X}: J\left(x ; \mathcal{L}^{*} \tau(x)\right)=u(x)\right\}
\end{aligned}
$$

are the stopping region, the continuation region, and the indifference region, respectively.

Remark 2.2 (Non-Existence of Optimal Stopping Times). The way we formulate timeconsistency in Definition [2.1, which follows a long thread of literature in Economics and Mathematical Finance, hinges on the existence of (pre-committed) optimal controls/stopping times at every state. When an optimal strategy fails to exist, it is unclear how to define timeconsistency. Recently, Karnam, Ma and Zhang (2016) point out this problem, and propose a possible way to formulate time-consistency, without referring to optimal strategies, via backward stochastic different equations (BSDEs). However, our game-theoretic approach described above - which eventually leads to a sophisticated stopping strategy - does not rely on the existence of optimal strategies.

Given any arbitrarily given stopping law $\tau \in \mathcal{T}(\mathbb{X})$, the above game-theoretic thinking gives rise to an alternative stopping law, $\Theta \tau$, which is no worse than $\tau$ to this sophisticated agent. Naturally, an equilibrium stopping law can be defined as one that is invariant under such a game-theoretic reasoning. This motivates Definition 2.4 below.

However, to carry this idea through, we need to identify conditions for the objective functional $J$ under which $\Theta \tau$ is indeed a stopping law satisfying the measurability requirement per Definition 2.2.

To this end, for any $\tau: \mathbb{X} \mapsto\{0,1\}$, we consider the kernel of $\tau$, which is the collection of states at which the agent stops, defined by

$$
\operatorname{ker}(\tau):=\{x \in \mathbb{X}: \tau(x)=0\} .
$$

Remark 2.3. For any $\tau \in \mathcal{T}(\mathbb{X})$ and $x \in \mathbb{X}, \mathcal{L} \tau(x)$ and $\mathcal{L}^{*} \tau(x)$ belong to $\mathcal{T}$. Indeed, the measurability of $\tau \in \mathcal{T}(\mathbb{X})$ implies

$$
\operatorname{ker}(\tau) \in \mathcal{B}(\mathbb{X})
$$

Thanks to the right continuity of the filtration $\mathbb{F}$,

$$
\mathcal{L} \tau(x)=\inf \left\{t \geq 0: X_{t}^{x} \in \operatorname{ker}(\tau)\right\} \quad \text { and } \quad \mathcal{L}^{*} \tau(x)=\inf \left\{t>0: X_{t}^{x} \in \operatorname{ker}(\tau)\right\}
$$

are $\mathbb{F}$-stopping times.

Now we introduce the assumptions that will ensure the measurability of $\Theta \tau$.

Assumption 2.1. The objective function $J:(\mathbb{X}, \mathcal{T}) \mapsto \mathbb{R}$ satisfies

(i) for any $D \in \mathcal{B}(\mathbb{X})$, the map $x \mapsto J\left(x ; T_{D}^{x}\right)$ is Borel measurable, where

$$
T_{D}^{x}:=\inf \left\{t>0: X_{t}^{x} \in D\right\} .
$$


(ii) for any sequence $\left\{D_{n}\right\}_{n \in \mathbb{N}}$ in $\mathcal{B}(\mathbb{X})$ such that $D_{n} \subseteq D_{n+1}$ for all $n \in \mathbb{N}$,

$$
\liminf _{n \rightarrow \infty} J\left(x ; T_{D_{n}}^{x}\right) \leq J\left(x ; T_{D}^{x}\right), \quad \text { where } D:=\cup_{n \in \mathbb{N}} D_{n} .
$$

Note that under the standard expected payoff formulation in (2.2), condition (i) above is true by definition, while condition (ii) holds under certain growth conditions on $u$ or on the process $X$, which depend on the specific problem at hand.

Proposition 2.1. Suppose Assumption 2.1 holds. Then $\Theta \tau \in \mathcal{T}(\mathbb{X})$ whenever $\tau \in \mathcal{T}(\mathbb{X})$.

Proof. In view of (2.9), $\mathcal{L}^{*} \tau(x)$ is simply $T_{\operatorname{ker}(\tau)}^{x}$. Thus, by Assumption 2.1(i), $x \mapsto J\left(x ; \mathcal{L}^{*} \tau(x)\right)$ is Borel measurable, whence $S_{\tau}, C_{\tau}$, and $I_{\tau}$ are all in $\mathcal{B}(\mathbb{X})$. By (2.7) and (2.8),

$$
\operatorname{ker}(\Theta \tau)=S_{\tau} \cup\left(I_{\tau} \cap \operatorname{ker}(\tau)\right) \in \mathcal{B}(\mathbb{X}),
$$

which implies that $\Theta \tau: \mathbb{X} \mapsto\{0,1\}$ is Borel measurable.

Definition 2.4 (Equilibrium (Sophisticated) Stopping Law). A stopping law $\tau \in \mathcal{T}(\mathbb{X})$ is called an equilibrium if $\Theta \tau(x)=\tau(x)$ for all $x \in \mathbb{X}$. We denote by $\mathcal{E}(\mathbb{X})$ the collection of all equilibrium stopping laws.

Remark 2.4 (Trivial Equilibrium). As with almost all the Nash-type equilibria, existence and uniqueness are important problems. In our setting, a stopping law $\tau \in \mathcal{T}(\mathbb{X})$ defined by $\tau(x)=0$ for all $x \in \mathbb{X}$ is trivially an equilibrium. Indeed, for any $x \in \mathbb{X}, \mathcal{L}^{*} \tau(x)=0$ and thus $J\left(x ; \mathcal{L}^{*} \tau(x)\right)=u(x)$. This implies $I_{\tau}=\mathbb{X}$. By (2.7), we conclude $\Theta \tau(x)=\tau(x)$ for all $x \in \mathbb{X}$.

To search for equilibrium stopping laws, the general (and natural) idea is to perform fixed-point iterations on the operator $\Theta$ : starting from any $\tau \in \mathcal{T}(\mathbb{X})$, take

$$
\tau_{*}(x):=\lim _{n \rightarrow \infty} \Theta^{n} \tau(x) \quad x \in \mathbb{X} .
$$

The above procedure admits a clear economic interpretation. At first, the (sophisticated) agent has an initial stopping law $\tau$. Once he starts to carry out the game-theoretic reasoning stipulated earlier, he realizes that the best stopping strategy for him, given that all future selves will follow $\tau$, is $\Theta \tau$. He therefore switches from $\tau$ to $\Theta \tau$. The same game-theoretic reasoning then implies that the best stopping strategy for him, given that all future selves will follow $\Theta \tau$, is $\Theta^{2} \tau$. The agent thus switches again from $\Theta \tau$ to $\Theta^{2} \tau$. This procedure continues until the agent eventually arrives at an equilibrium $\tau_{*}$, a fixed point of $\Theta$ and a strategy he cannot further improve upon by the procedure just described. In economic terms, each application of $\Theta$ corresponds to an additional level of strategic reasoning.

Mathematically, we need to prove that the limit taken in (2.11) is well-defined, belongs to $\mathcal{T}(\mathbb{X})$, and satisfies $\Theta \tau_{*}=\tau_{*}$. In general, such results are not easy to establish, and remain largely an open question.

\footnotetext{
${ }^{7}$ For a stopping problem with expected utility and non-exponential discounting, Huang and Nguven-Huu (2016) show that fixed-point iterations do converge to equilibriums, under appropriate conditions on the discount function and the initial stopping law $\tau \in \mathcal{T}(\mathbb{X})$; see Theorem 3.1 and Corollary 3.1 therein.
} 
However, when $X$ is a one-dimensional diffusion process we will be able to derive the stopping strategies of the naïvités and the sophisticates in a fairly complete and explicit manner. This is not only because, in the one-dimensional case, pre-committed stopping laws have been obtained rather thoroughly by Xu and Zhou (2013) on which a naïve strategy depends, but also because the fixed-point iteration (2.11) turns out to be much more manageable and does converge to an equilibrium, due to a key technical result (Lemma 3.1) that holds only for a one-dimensional process.

\section{Equilibrium Stopping Laws: The One-Dimensional Case}

Suppose $\mathbb{X} \subseteq \mathbb{R}$. Let $X$ be a one-dimensional stationary diffusion process satisfying the dynamics

$$
d X_{t}=b\left(X_{t}\right) d t+a\left(X_{t}\right) d B_{t}, \quad X_{0}=x \in \mathbb{X},
$$

where $B$ is a standard one-dimensional Brownian motion, and $a, b$ are real-valued Borel measurable functions with $a(\cdot)>0$. Define $\theta(\cdot):=b(\cdot) / a(\cdot)$, and assume that

$$
\mathbb{P}\left[\int_{0}^{t} \theta^{2}\left(X_{s}\right) d s<\infty\right]=1, \quad \forall t \geq 0 .
$$

Introduce the process

$$
Z_{t}:=\exp \left(-\int_{0}^{t} \theta\left(X_{s}\right) d B_{s}-\frac{1}{2} \int_{0}^{t} \theta^{2}\left(X_{s}\right) d s\right) \quad \text { for } t \geq 0,
$$

which is by definition a nonnegative local martingale. Note that (3.1) is a standard condition

ensuring that $\int_{0}^{t} \theta\left(X_{s}\right) d B_{s}$ is well-defined for all $t \geq 0$ a.s.. Consider the first revisit time to the initial value $x$ :

$$
T_{x}^{x}:=\inf \left\{t>0: X_{t}^{x}=x\right\} \in \mathcal{T} .
$$

We also introduce the running maximum and minimum processes defined respectively by

$$
\bar{X}_{t}^{x}:=\max _{s \in[0, t]} X_{s}^{x} \quad \text { and } \quad \underline{X}_{t}^{x}:=\min _{s \in[0, t]} X_{s}^{x}, \quad t \geq 0 .
$$

Lemma 3.1. Suppose (3.1) holds and $Z$ defined in (3.2) is a martingale. Then, for any $x \in \mathbb{X}, \mathbb{P}\left[\bar{X}_{t}^{x}>x\right]=\mathbb{P}\left[\underline{X}_{t}^{x}<x\right]=1$ for all $t>0$. Hence, $T_{x}^{x}=0$ a.s.

The proof of this lemma is supplied in Appendix $\mathrm{A}$.

Proposition 3.1. Suppose $\mathbb{X} \subseteq \mathbb{R}$, Assumption 2.1 holds, and $Z$ defined in (3.2) is a martingale. Then, for any $\tau \in \mathcal{T}(\mathbb{X})$,

$$
\begin{aligned}
& \operatorname{ker}(\tau) \subseteq I_{\tau}, \\
\operatorname{ker}\left(\Theta^{n} \tau\right)= & S_{\Theta^{n-1} \tau} \cup \operatorname{ker}\left(\Theta^{n-1} \tau\right), \quad \forall n \in \mathbb{N} .
\end{aligned}
$$

Hence, $\tau_{*}$ in (2.11) is well-defined, with $\operatorname{ker}\left(\tau_{*}\right)=\bigcup_{n \in \mathbb{N}} \operatorname{ker}\left(\Theta^{n} \tau\right)$. 
Proof. For any $x \in \operatorname{ker}(\tau)$, there are three possible cases:

1. $x$ is an interior point of $\operatorname{ker}(\tau)$ : Then $\mathcal{L}^{*} \tau(x)=0$ by definition, and thus $J\left(x ; \mathcal{L}^{*} \tau(x)\right)=$ $u(x)$, i.e. $x \in I_{\tau}$.

2. $x$ is a boundary point of $\operatorname{ker}(\tau)$ : By Lemma $3.1, \mathbb{P}\left[\bar{X}_{t}^{x}>x\right]=\mathbb{P}\left[\underline{X}_{t}^{x}<x\right]=1$ for all $t>0$. This implies $\mathcal{L}^{*} \tau(x)=\inf \left\{t>0: X_{t}^{x} \in \operatorname{ker}(\tau)\right\}<1 / n$ for all $n \in \mathbb{N}$ a.s., and thus $\mathcal{L}^{*} \tau(x)=0$ a.s. It follows that $J\left(x ; \mathcal{L}^{*} \tau(x)\right)=u(x)$, i.e. $x \in I_{\tau}$.

3. $x$ is an isolated point of $\operatorname{ker}(\tau)$, i.e. $\exists \varepsilon>0$ such that $(x-\varepsilon, x+\varepsilon) \cap \operatorname{ker}(\tau)=\{x\}$ : Since $T_{x}^{x}=0$ a.s. (by Lemma 3.1), $\mathcal{L}^{*} \tau(x)=0$ a.s. This gives $J\left(x ; \mathcal{L}^{*} \tau(x)\right)=u(x)$, i.e. $x \in I_{\tau}$.

It follows that $\operatorname{ker}(\tau) \subseteq I_{\tau}$. This, together with (2.7), shows that $\operatorname{ker}(\Theta \tau)=S_{\tau} \cup\left(I_{\tau} \cap\right.$ $\operatorname{ker}(\tau))=S_{\tau} \cup \operatorname{ker}(\tau)$. By repeating the same argument above for each $n>1$, we obtain (3.4).

Since $\left\{\operatorname{ker}\left(\Theta^{n} \tau\right)\right\}_{n \in \mathbb{N}}$ is a nondecreasing sequence of Borel sets, if $x \in \bigcup_{n \in \mathbb{N}} \operatorname{ker}\left(\Theta^{n} \tau\right)$, then there exists $N>0$ such that $\Theta^{n} \tau(x)=0$ for all $n \geq N$; if $x \notin \bigcup_{n \in \mathbb{N}} \operatorname{ker}\left(\Theta^{n} \tau\right)$, then $\Theta^{n} \tau(x)=1$ for all $n \in N$. This already implies that the limit-taking in (2.11) is well-defined, and $\operatorname{ker}\left(\tau_{*}\right)=\bigcup_{n \in \mathbb{N}} \operatorname{ker}\left(\Theta^{n} \tau\right)$.

Theorem 3.1. Suppose $\mathbb{X} \subseteq \mathbb{R}$, Assumption 2.1 holds, and $Z$ defined in (3.2) is a martingale. Then, for any $\tau \in \mathcal{T}(\mathbb{X}), \tau_{*}$ defined in (2.11) belongs to $\mathcal{E}(\mathbb{X})$. Hence,

$$
\mathcal{E}(\mathbb{X})=\left\{\tau_{*} \in \mathcal{T}(\mathbb{X}): \tau_{*}=\lim _{n \rightarrow \infty} \Theta^{n} \tau, \text { for some } \tau \in \mathcal{T}(\mathbb{X})\right\}=\bigcup_{\tau \in \mathcal{T}(\mathbb{X})}\left\{\lim _{n \rightarrow \infty} \Theta^{n} \tau\right\}
$$

Proof. By Proposition [3.1, $\tau_{*} \in \mathcal{T}(\mathbb{X})$ is well-defined and $\operatorname{ker}\left(\tau_{*}\right)=\bigcup_{n \in \mathbb{N}} \operatorname{ker}\left(\Theta^{n} \tau\right)$. Since $\operatorname{ker}\left(\tau_{*}\right) \subseteq I_{\tau_{*}}$ (by Proposition 3.1 again), $\Theta \tau_{*}(x)=\tau_{*}(x)$ for all $x \in \operatorname{ker}\left(\tau_{*}\right)$. For $x \notin$ $\operatorname{ker}\left(\tau_{*}\right)$, we have $x \notin \operatorname{ker}\left(\Theta^{n} \tau\right)$, i.e. $\Theta^{n} \tau(x)=1$, for all $n \in \mathbb{N}$. In view of (2.7), this gives $J\left(x ; \mathcal{L}^{*} \Theta^{n-1} \tau(x)\right) \geq u(x)$ for all $n \in \mathbb{N}$. By (2.9), this can be written as $J\left(x ; T_{\operatorname{ker}\left(\Theta^{n-1} \tau\right)}^{x}\right) \geq$ $u(x)$ for all $n \in \mathbb{N}$. Thanks to Proposition 3.1, $\left\{\operatorname{ker}\left(\Theta^{n-1} \tau\right)\right\}_{n \in \mathbb{N}}$ is a nondecreasing sequence of Borel sets and $\operatorname{ker}\left(\tau_{*}\right)=\bigcup_{n \in \mathbb{N}} \operatorname{ker}\left(\Theta^{n-1} \tau\right)$. It then follows from Assumption 2.1 (ii) that

$$
J\left(x ; T_{\operatorname{ker}\left(\tau_{*}\right)}^{x}\right) \geq \liminf _{n \rightarrow \infty} J\left(x ; T_{\operatorname{ker}\left(\Theta^{n-1} \tau\right)}^{x}\right) \geq u(x) .
$$

This implies $x \in C_{\tau_{*}} \cup I_{\tau_{*}}$. If $x \in I_{\tau_{*}}$, then $\Theta \tau_{*}(x)=\tau_{*}(x)$; if $x \in C_{\tau_{*}}$, then $\Theta \tau_{*}(x)=1=$ $\tau_{*}(x)$, as $x \notin \operatorname{ker}\left(\tau_{*}\right)$. Thus, we conclude that $\Theta \tau_{*}(x)=\tau_{*}(x)$ for all $x \in \mathbb{X}$, i.e. $\tau_{*} \in \mathcal{E}(\mathbb{X})$.

For the first equality in (3.5), the " $\supseteq$ " relation holds as a direct consequence of the above result. Note that the "ᄃ" relation is also true because for each $\tau_{*} \in \mathcal{E}(\mathbb{X})$, one can simply take $\tau \in \mathcal{T}(\mathbb{X})$ on the right hand side to be $\tau_{*}$ itself. The last set in (3.5) is simply a re-formulation of the second set.

This theorem suggests that under its assumptions every equilibrium can be found through the fixed-point iteration. Moreover, it stipulates a way of telling whether a given stopping law is an equilibrium. Any initial strategy that can be strictly improved by the iteration (namely the game-theoretic reasoning) is not an equilibrium strategy. On the other hand, 
even if the agent's initial strategy happens to be already an equilibrium, he may not realize it. He does only after he has applied the iteration and found that he gets the same strategy.

Any given stopping law $\tau$ will give rise to an equilibrium $\tau_{*}$ according to (3.5); so in general it is unlikely that we will have a unique equilibrium. In this paper, we are particularly interested in the equilibrium $\hat{\tau}_{*} \in \mathcal{E}(\mathbb{X})$ generated by the naïve stopping law $\hat{\tau} \in \mathcal{T}(\mathbb{X})$ which is induced by $\left\{\hat{\tau}_{x}\right\}_{x \in \mathbb{X}}$ in $(2.5)$; that is,

$$
\hat{\tau}_{*}(x)=\lim _{n \rightarrow \infty} \Theta^{n} \hat{\tau}(x) \quad x \in \mathbb{X} .
$$

The economic significance of choosing such an equilibrium is that it spells out explicitly how an (irrational) naïve agent might be turned into a (fully rational) sophisticated one, if he is educated to carry out sufficient levels of strategic reasoning.

\section{Probability Distortion: Time (In)consistency}

In the remainder of this paper we apply the general setting and results established in the previous section to the case where the objective functional involves probability distortion (weighting) and the underlying process is a geometric Brownian motion. This section is devoted to categorizing several cases and their time (in)consistency.

Let $S=\left\{S_{t}\right\}_{t \geq 0}$ be a geometric Brownian motion satisfying

$$
d S_{t}=\mu S_{t} d t+\sigma S_{t} d B_{t}, \quad S_{0}=s>0, \quad \text { for } t \geq 0,
$$

where $\mu \in \mathbb{R}$ and $\sigma>0$ are constants and $\left\{B_{t}\right\}_{t \geq 0}$ is a standard Brownian motion. Note that the process $S$ takes values in $\mathbb{R}_{+}:=(0, \infty)$ almost surely. In most of the discussions in this paper, $S$ is interpreted as the price process of an asset, although it could represent other processes such as the value process of a project and similar discussions could be conducted.

Consider a nondecreasing, continuous function $U: \mathbb{R}_{+} \mapsto \mathbb{R}_{+}$with $U(0)=0$, and a strictly increasing, continuous function $w:[0,1] \mapsto[0,1]$ with $w(0)=0$ and $w(1)=1$. Here, $U$ is the utility function of the agent or the payoff function of the asset, and $w$ the probability distortion function. The agent's objective is, for any given initial state $s$, to maximize his "distorted" expected payoff:

$$
\operatorname{maximize} \quad I(s ; \tau):=\int_{0}^{\infty} w\left(\mathbb{P}\left[U\left(S_{\tau}^{s}\right)>y\right]\right) d y,
$$

by choosing an appropriate stopping time $\tau \in \mathcal{T}$.

So the agent intends to maximize his expected utility (or payoff) under probability distortion, by stopping the price process at an appropriate moment. This formulation is motivated by several financial applications, such as liquidation of a financial position, real options and casino gambling. Note that with $w(p)=p$, we retrieve the standard expectation valuation.

In the rest of the paper, we denote

$$
\beta:=1-2 \mu / \sigma^{2}
$$

We will show below that the sign of $\beta$ critically affects the time-consistency of the underlying problem. 


\subsection{The Case $\beta=0$}

When $\beta=0$ (i.e. $\left.\mu=\frac{1}{2} \sigma^{2}\right), S_{t}=s e^{\sigma B_{t}}$ for $t \geq 0$. If the $\operatorname{supremum} \sup \{U(x): x>0\}$ is not attained, then $\mathrm{Xu}$ and Zhou (2013), p. 255, shows that an optimal stopping time of (4.2) does not exist. In this case, the naïve agent never stops; recall (2.5).

If the supremum $\sup \{U(x): x>0\}$ is attained, let $x^{*}:=\inf \left\{x>0: U(x)=\sup _{y>0} U(y)\right\}$. Then $\mathrm{Xu}$ and Zhou (2013), p. 255, proves that

$$
\hat{\tau}_{s}:=\inf \left\{t \geq 0: S_{t}^{s} \geq x^{*}\right\}=\inf \left\{t \geq 0: B_{t} \geq \sigma^{-1} \log \left(x^{*} / s\right)\right\}
$$

is a (pre-committed) optimal stopping time of (4.2). The stopping threshold for the state process $S^{s}$ is always $x^{*}$, independent of the current state $s$. There is then no time-inconsistency here. Indeed, for any $t>0$ and $s>0$, if $\hat{\tau}_{s}>t$, then $\hat{\tau}_{s}=\inf \left\{r \geq t: S_{r}^{s} \geq x^{*}\right\}=t+\hat{\tau}_{S_{t}^{s}}$; that is, (2.3) is satisfied. In this case, the three types of agents behave identically.

\subsection{The Case $\beta \neq 0$ : Transformation}

When $\beta \neq 0$, we consider, as in Section 2.2 of $\mathrm{Xu}$ and Zhou $(2013)$, the process $X_{t}:=S_{t}^{\beta}$, $t \geq 0$. That is, $X$ is the Doléans-Dade exponential of $\left\{\beta \sigma B_{t}\right\}_{t \geq 0}$ :

$$
d X_{t}^{x}=\beta \sigma X_{t}^{x} d B_{t}, \quad X_{0}^{x}=x>0,
$$

where $x=s^{\beta}>0$. As a geometric Brownian motion with no drift, $X^{x}$ is a $\mathbb{P}$-martingale and

$$
\lim _{t \rightarrow \infty} X_{t}^{x}=0 \quad \text { a.s. }
$$

thanks to the law of iterated logarithms. As we will see subsequently, this fact plays an important role in dictating a sophisticated agent's behavior - the value of the underlying process diminishes in the long run. Moreover, for any $b>x$,

$$
\mathbb{P}\left[T_{b}^{x}<\infty\right]=\frac{x}{b}<1, \quad \text { where } \quad T_{b}^{x}:=\inf \left\{t>0: X_{t}^{x}=b\right\} .
$$

Note that (4.7) follows from the hitting time probability of a standard Brownian motion to a linear boundary; see e.g. Karatzas and Shreve (1991).

Let $u$ be defined by

$$
u(x):=U\left(x^{1 / \beta}\right), \quad x \in(0, \infty) .
$$

Note that the transformed function $u$ is nondecreasing if $\beta>0$ and nonincreasing if $\beta<0$. The shape of $u$, which can be convex, concave, or $S$-shaped (i.e. first convex, and then concave), depends on both the shape of $U$ and the coefficient $\beta$.

Remark 4.1 (Goodness Index). The value of $\beta$, the shape of $u$, and how "good" the asset $S$ is (in terms of its risk-return profile) are all inter-connected. Shiryaev, Xu and Zhou (2008) take $\frac{\mu}{\sigma^{2}}$ as the "goodness index" of an asset, which is included in our definition of $\beta$ in (4.3). The larger this index is, the higher the asset's expected return relative to its volatility. More specifically, Section 2.3 of Xu and Zhou (2013) discusses several concrete examples, which leads to the following general observations: when $\beta<0$ (or $\frac{\mu}{\sigma^{2}}>\frac{1}{2}$ ), the asset $S$ is considered "good", and $u$ in this case is usually convex; as $\beta$ increases (or $\frac{\mu}{\sigma^{2}}$ decreases), the asset $S$ changes gradually to "average" and then to "bad", with u changing gradually to "S-shaped" and then to "concave". 
Similarly, we define

$$
J(x ; \tau):=I\left(x^{1 / \beta} ; \tau\right) \equiv \int_{0}^{\infty} w\left(\mathbb{P}\left[u\left(X_{\tau}^{x}\right)>y\right]\right) d y \quad \text { for } x>0, \tau \in \mathcal{T} .
$$

Here, we allow $\tau \in \mathcal{T}$ to take the value $+\infty$ with positive probability: on the set $\{\tau=\infty\}$, we simply take $X_{\tau}=0$ in view of (4.6). As shown in Lemma 3.1 of Xu and Zhou (2013), for any $\tau \in \overline{\mathcal{T}}:=\{\tau \in \mathcal{T} \mid \mathbb{P}[\tau<\infty]=1\}$, (4.9) admits the following expressions:

$$
\begin{aligned}
& J(x ; \tau)=J_{D}(x ; F):=\int_{0}^{\infty} w(1-F(y)) u^{\prime}(y) d y, \\
& J(x ; \tau)=J_{Q}(x ; G):=\int_{0}^{1} u(G(y)) w^{\prime}(1-y) d y,
\end{aligned}
$$

where $F$ is the cumulative distribution function (CDF) of $X_{\tau}^{x}$, and $G:=F^{-1}$ is the quantile function of $X_{\tau}^{x}$. Moreover,

$$
\sup _{\tau \in \overline{\mathcal{T}}} J(x ; \tau)=\sup _{F \in \mathcal{D}} J_{D}(x ; F)=\sup _{G \in \mathcal{Q}} J_{Q}(x ; G),
$$

where

$$
\begin{aligned}
& \mathcal{D}:=\left\{F: \mathbb{R}_{+} \mapsto[0,1] \mid F \text { is a CDF of } X_{\tau}^{x}, \text { for some } \tau \in \mathcal{T}\right\} \\
& \mathcal{Q}:=\left\{G:[0,1) \mapsto \mathbb{R}_{+} \mid G=F^{-1} \text { for some } F \in \mathcal{D}\right\} .
\end{aligned}
$$

By Proposition B.1, the equivalence relation (4.12) can be extended from $\overline{\mathcal{T}}$ to $\mathcal{T}$, i.e.

$$
\sup _{\tau \in \mathcal{T}} J(x ; \tau)=\sup _{F \in \mathcal{D}} J_{D}(x ; F)=\sup _{G \in \mathcal{Q}} J_{Q}(x ; G) .
$$

Remark 4.2 (Naïve Law via Distribution or Quantile). The naïve stopping law $\hat{\tau} \in \mathcal{T}\left(\mathbb{R}_{+}\right)$ induced by optimal stopping times $\left\{\hat{\tau}_{x}\right\}_{x \in \mathbb{R}_{+}}$(see (2.5)) can also be expressed using the distribution or quantile function, $F_{\hat{\tau}_{x}}$ or $G_{\hat{\tau}_{x}}$ respectively, of $X_{\hat{\tau}_{x}}^{x}$. That is,

$$
\hat{\tau}(x):=\left\{\begin{array}{l}
0, \text { if } \hat{\tau}_{x}=0, \\
1, \text { if otherwise. }
\end{array}=\left\{\begin{array}{l}
0, \text { if } F_{\hat{\tau}_{x}}(\cdot)=1_{[x, \infty)}(\cdot), \\
1, \text { if otherwise. }
\end{array} \quad=\left\{\begin{array}{l}
0, \text { if } G_{\hat{\tau}_{x}}(\cdot) \equiv x, \\
1, \text { if otherwise. }
\end{array}\right.\right.\right.
$$

\subsection{The Case $\beta<0$}

When $\beta<0, u$ defined in (4.8) is nonincreasing with $u(0+)=U(\infty)$ and $u(\infty)=U(0+)$. By Theorem 2.1 in $\mathrm{Xu}$ and Zhou (2013), $\sup _{\tau \in \mathcal{T}} J(x ; \tau)=u(0+)$ and $\hat{\tau}_{x} \equiv \infty$, implying "never-stopping", is the optimal stopping time. Again, no time-inconsistency is present here as $\hat{\tau}_{x} \equiv \infty$ does not depend on current state $x$, and all the three types of agents do not stop in any circumstances. The economic interpretation is that, in the context of selling an asset, $\beta<0$ corresponds to a "good asset", and hence all the agents hold the asset perpetually in the spirit of Warren Buffet's "buy and hold a good asset". See also Xu and Zhou (2013), p. 257 , for a discussion. 


\subsection{The Case $\beta>0$ : Time-Inconsistency}

When $\beta>0, u$ defined in (4.8) is nondecreasing with $u(0)=U(0)=0$. Under the objective functional (4.9), $\mathrm{Xu}$ and Zhou (2013) characterize the pre-committed optimal stopping times for problem (2.1), noting that the problem may be time-inconsistent as $\beta>0$. The next example demonstrates this time-inconsistency explicitly.

Example 4.1. Take $u(x)=x^{\eta}$ with $\eta \geq 1$, and consider the probability weighting function proposed by Preled (1998):

$$
w(x)=\exp \left(-\gamma(-\log x)^{\alpha}\right) \quad \text { for some } \alpha, \gamma>0 .
$$

Since $u$ is convex, Theorem 4.5 of $\mathrm{Xu}$ and Zhou (2013) shows that problem (2.1), with $J(x ; \tau)$ as in (4.9), can be reduced to the optimization problem

$$
\sup _{\lambda \in(0,1]} w(\lambda) u\left(\frac{x}{\lambda}\right)
$$

To solve this problem for our case, for each $x>0$, set $f(\lambda):=w(\lambda) u\left(\frac{x}{\lambda}\right)$. By direct computation,

$$
f^{\prime}(\lambda)=\frac{w(\lambda)}{\lambda}\left(\frac{x}{\lambda}\right)^{\eta}\left[\alpha \gamma(-\log \lambda)^{\alpha-1}-\eta\right]
$$

Observe that $f^{\prime}(\lambda)=0$ has a unique solution $\lambda^{*}=e^{-\left(\frac{\eta}{\alpha \gamma}\right)^{1 /(\alpha-1)}}$ on $(0,1]$. Moreover,

$$
f^{\prime \prime}\left(\lambda^{*}\right)=-\alpha \gamma(\alpha-1)\left(\frac{\eta}{\alpha \gamma}\right)^{\frac{\alpha-2}{\alpha-1}} \frac{w\left(\lambda^{*}\right)}{\left(\lambda^{*}\right)^{2}}\left(\frac{x}{\lambda^{*}}\right)^{\eta} .
$$

Suppose $\alpha>1$, in which case $w$ is $S$-shaped (i.e. first convex, and then concave). Then $f^{\prime \prime}\left(\lambda^{*}\right)<0$; moreover, $f^{\prime}(\lambda)>0$ for $\lambda<\lambda^{*}$, and $f^{\prime}(\lambda)<0$ for $\lambda>\lambda^{*}$. This implies that $\lambda^{*}$ is the unique maximizer of (4.16). By the discussion following Corollary 4.6 in Xu and Zhou (2013), we conclude that

$$
\hat{\tau}_{x}:=\inf \left\{t \geq 0: X_{t}^{x} \geq x / \lambda^{*}\right\}=\inf \left\{t \geq 0: X_{t}^{x} \geq e^{\left(\frac{\eta}{\alpha \gamma}\right)^{1 /(\alpha-1)}} x\right\} \in \mathcal{T}
$$

is a pre-committed optimal stopping time of (2.1) when the current state is $x$. A moving stopping threshold causes time-inconsistency: for any $t>0, \hat{\tau}_{x} \neq t+\hat{\tau}_{X_{t}^{x}}$ on $\left\{\hat{\tau}_{x}>t\right\}$, unless $X_{t}^{x} \equiv x$; thus, (2.3) is in general violated. More specifically, as $X$ evolves over time, the agent continuously updates the initial value $x$ in (2.1) with current state $y:=X_{t}$, and thus changes the original stopping threshold $x / \lambda^{*}$ to $y / \lambda^{*}$ at time $t$. While being an optimal solution to (2.1) at state $x, \hat{\tau}_{x}$ will not be implemented as future selves will dismiss it.

A crucial observation here is that time-inconsistency leads the naïve agent to postpone stopping indefinitely. Since $1 / \lambda^{*}>1$, we have $\hat{\tau}_{x}>0$ for all $x>0$, whence the naïve agent will never stop at any given moment and - as a result - will never stop. 
The above example is reminiscent of Ebert and Strack (2015), which shows - under the CPT setting - that a naïve agent may defer his stopping decision indefinitely (hence disobey the original pre-committed optimal stopping time) under the so-called "skewness preference in the small" assumption 8

Example 4.2. Take $u(x)=(x-K)^{+}$for some $K>0$ and $w(x)=\eta x^{2}+(1-\eta) x$ for some $\eta \in(0,1)$. In this case both $u$ and $w$ are convex. Then (4.16) becomes $\sup _{\lambda \in(0,1]} f(\lambda)$ with $f(\lambda):=\left(\eta \lambda^{2}+(1-\eta) \lambda\right)\left(\frac{x}{\lambda}-K\right)^{+}$. For $x>K, f^{\prime}(\lambda)=\eta x-(1-\eta) K-2 \eta K \lambda$. Define

$$
\bar{\lambda}:=\frac{\eta(x+K)-K}{2 \eta K} \text {. }
$$

If $x>\frac{\eta+1}{\eta} K, f^{\prime}(\lambda)>(\eta+1) K-(1-\eta) K-2 \eta K=0$ for all $\lambda \in(0,1]$, which implies that $\lambda^{*}=1$ is the maximizer. If $K<x \leq \frac{\eta+1}{\eta} K$, then $\lambda^{*}=\bar{\lambda} \leq 1$ is the maximizer. For $x \leq K$, observe that

$$
f(\lambda)= \begin{cases}-\eta K[\lambda-\bar{\lambda}]^{2}+(1-\eta) x+\eta K \bar{\lambda}^{2}, & \text { if } 0<\lambda \leq \frac{x}{K}, \\ 0, & \text { if } \frac{x}{K}<\lambda \leq 1 .\end{cases}
$$

If $\eta \leq 1 / 2$, then $\bar{\lambda} \leq \frac{x-K}{4 \eta K} \leq 0$. This, together with (4.18), implies that $\sup _{\lambda \in(0,1]} f(\lambda)$ admits no maximizer. If $\eta>1 / 2$, then for $x>\frac{1-\eta}{\eta} K$, it can be checked that $\bar{\lambda} \in\left(0, \frac{x}{K}\right)$, which implies that $\lambda^{*}=\bar{\lambda}$ is the maximizer (thanks again to (4.18) ); for $x \leq \frac{1-\eta}{\eta} K$, we have $\bar{\lambda} \leq 0$ and thus $\sup _{\lambda \in(0,1]} f(\lambda)$ admits no maximizer. Thus, we conclude that

$$
\hat{\tau}_{x}:= \begin{cases}\inf \left\{t \geq 0: X_{t}^{x} \geq x\right\}=0, & \text { if } x>\frac{\eta+1}{\eta} K, \\ \inf \left\{t \geq 0: X_{t}^{x} \geq \frac{2 \eta K x}{\eta(x+K)-K}\right\}>0, & \text { if }\left(\frac{1-\eta}{\eta} \wedge 1\right) K<x \leq \frac{\eta+1}{\eta} K,\end{cases}
$$

is a (pre-committed) optimal stopping time of (2.1) for $x>\left(\frac{1-\eta}{\eta} \wedge 1\right) K$, and there exists no optimal stopping time for $x \leq\left(\frac{1-\eta}{\eta} \wedge 1\right) K$. We observe time-inconsistency here: the stopping threshold $\frac{2 \eta K x}{\eta(x+K)-K}$ depends on current state $x$, when $\left(\frac{1-\eta}{\eta} \wedge 1\right) K<x \leq \frac{\eta+1}{\eta} K$.

The naïve stopping law is thus given by

$$
\hat{\tau}(x)=1_{\left(0, \frac{\eta+1}{\eta} K\right)}(x), \quad \text { for all } x>0,
$$

a threshold-type (e.g. stop-loss or stop-gain) stopping strategy.

It is interesting that in this example even a naïvité will stop, seemingly contradicting the result of Ebert and Strack 2015). However, there is really no contradiction - the convexity of $w$ violates Assumption 2 of Ebert and Strack (2015). In economics terms, a convex distortion overweights the small probabilities of very bad events and underweights those of very good events; so a small, right-skewed, lottery-like gamble is unattractive to the agent. As a result, he decides that he will stop once the process hits the threshold, $\frac{\eta+1}{\eta} K$. This example also suggests that RDU/CPT together with the time-inconsistent theory can indeed offer realistic predictions if we allow modeling of different types of preferences and different characteristics of the underlying process.

\footnotetext{
${ }^{8}$ Indeed Example 4.1 satisfies Assumptions 1 and 2 in Ebert and Strack (2015), which are sufficient for the "skewness preference in the small" to hold.
} 


\section{$5 \quad$ Equilibrium Stopping Law: The Case $\beta>0$}

In the previous section we have demonstrated in two examples that, when $\beta>0$, timeinconsistency arises. We have also derived the respective naïve strategies in the examples.

The next step is to study how a sophisticated agent lacking commitment might be doing by applying the general result Theorem 3.1. We are particularly interested in finding the equilibrium stopping law $\hat{\tau}_{*} \in \mathcal{E}\left(\mathbb{R}_{+}\right)$generated by the naïve stopping law, as defined in (3.6). Through this construction of an equilibrium we will be able to see an intriguing connection between a naïve agent and a sophisticated one - in particular how the former might turn himself into the latter by evoking strategic reasonings. Details of these will be presented in Section 6, and the current section is focused on developing the necessary theoretic foundations.

Technically, in order to apply Theorem 3.1 we need to check the validity of its assumptions. To this end, we need the following two results.

Lemma 5.1. Suppose $\beta>0$. For any $0 \leq a<x<b$, denote $\tau_{a b}:=\inf \left\{t \geq 0: X_{t}^{x} \notin\right.$ $(a, b)\} \in \mathcal{T}$.

(i) If $a=0$ and $b=\infty$, then $J\left(x ; \tau_{a b}\right)=0$;

(ii) If $a>0$ and $b=\infty$, then $J\left(x ; \tau_{a b}\right)=u(a)$;

(iii) If $a \geq 0$ and $b<\infty$, then

$$
J\left(x ; \tau_{a b}\right)=u(a)+w\left(\frac{x-a}{b-a}\right)(u(b)-u(a)) .
$$

The proof is relegated to Appendix C.

Lemma 5.2. Suppose $\beta>0$. The objective functional (4.9) satisfies Assumption 2.1.

The proof is deferred to Appendix D.

Theorem 5.1. Suppose $\beta>0$ and the objective functional is given by (4.9). For any $\tau \in \mathcal{T}\left(\mathbb{R}_{+}\right)$, $\tau_{*}$ defined in (2.11) belongs to $\mathcal{E}\left(\mathbb{R}_{+}\right)$, and hence $(3.5)$ holds with $\mathbb{X}=\mathbb{R}_{+}$. In particular, $\hat{\tau}_{*}$ defined by (3.6) belongs to $\mathcal{E}\left(\mathbb{R}_{+}\right)$.

Proof. Since $X$ is a geometric Brownian motion with zero drift, the process $Z$ in (3.2) is constantly 1 and thus a martingale. Since the objective functional (4.9) satisfies Assumption 2.1 (by Lemma 5.2), the result is now a direct consequence of Theorem 3.1 .

The following two examples demonstrate how a general initial stopping law $\tau \in \mathcal{T}\left(\mathbb{R}_{+}\right)$, which need not be the naïve law $\hat{\tau}$ in (2.5), converges to its corresponding equilibrium $\tau_{*} \in \mathcal{E}\left(\mathbb{R}_{+}\right)$.

Example 5.1. Let $\beta>0$, and take $u(x)=x$. This setup may generate time-inconsistency as Example 4.1 demonstrates. For arbitrary constants $0<a<b<\infty$, define the stopping law $\tau \in \mathcal{T}\left(\mathbb{R}_{+}\right)$by

$$
\tau(x):=1_{(a, b)}(x), \quad \text { for all } x>0 .
$$


Then $\mathcal{L}^{*} \tau(x)=\inf \left\{t>0 \mid X_{t}^{x} \notin(a, b)\right\}$. For $x \notin(a, b), \mathcal{L}^{*} \tau(x)=0$ and thus $J\left(x ; \mathcal{L}^{*} \tau(x)\right)=$ $u(x)$, which implies $(0, a] \cup[b, \infty) \subseteq I_{\tau}$. For $x \in(a, b)$, Lemma 5.1.(iii) indicates that

$$
J\left(x ; \mathcal{L}^{*} \tau(x)\right)-u(x)=(a-x)+w\left(\frac{x-a}{b-a}\right)(b-a) .
$$

If $w$ is concave and $w(x) \neq x$ for some $x>0$, then $w\left(\frac{x-a}{b-a}\right)>\frac{x-a}{b-a}$ for all $x \in(a, b)$. In view of (5.2), we have $C_{\tau}=(a, b)$. It follows that $\Theta \tau(x)=1_{(a, b)}(x)=\tau(x)$ for all $x>0$. That is, $\tau$ is itself an equilibrium stopping law, and thus $\tau_{*}=\tau$. Incidentally, it follows that there are infinitely many equilibrium stopping laws in this case.

If $w$ is convex and $w(x) \neq x$ for some $x>0$, then $w\left(\frac{x-a}{b-a}\right)<\frac{x-a}{b-a}$ for all $x \in(a, b)$, and thus $S_{\tau}=(a, b)$, thanks to (5.2). It follows that $\Theta \tau(x)=0$ for all $x>0$. That is, $\tau_{*}=\Theta \tau \equiv 0$, the trivial equilibrium that stops immediately.

Finally, consider the case when $w$ is inverse $S$-shaped and intersects $u(x)=x$ at some point $z^{*} \in(0,1)$. For any $x \in(a, b)$, observe that whether $w\left(\frac{x-a}{b-a}\right)$ is larger than, equal to, or less than $\frac{x-a}{b-a}$ depends on whether $\frac{x-a}{b-a}$ is less than, equal to, or large than $z^{*}$. It then follows from (5.2) that

$$
S_{\tau}=\left(a+z^{*}(b-a), b\right), \quad C_{\tau}=\left(a, a+z^{*}(b-a)\right), \quad \text { and } \quad a+z^{*}(b-a) \in I_{\tau} .
$$

By (3.4) $), \operatorname{ker}(\Theta \tau)=\operatorname{ker}(\tau) \cup S_{\tau}=(0, a] \cup\left(a+z^{*}(b-a), \infty\right)$, and thus $\Theta \tau(x)=1_{\left(a, a+z^{*}(b-a)\right]}(x)$ for all $x>0$. By the same argument as above, $S_{\Theta \tau}=\left(a+\left(z^{*}\right)^{2}(b-a), a+z^{*}(b-a)\right)$ and

$$
\operatorname{ker}\left(\Theta^{2} \tau\right)=\operatorname{ker}(\Theta \tau) \cup S_{\Theta \tau}=(0, a] \cup\left(a+\left(z^{*}\right)^{2}(b-a), a+z^{*}(b-a)\right) \cup\left(a+z^{*}(b-a), \infty\right) .
$$

In general, for each $n \in \mathbb{N}$,

$$
\operatorname{ker}\left(\Theta^{n} \tau\right)=(0, a] \cup\left(b_{n}, b_{n-1}\right) \cup\left(b_{n-1}, b_{n-2}\right) \cup \ldots \cup\left(b_{2}, b_{1}\right) \cup\left(b_{1}, \infty\right),
$$

where $b_{k}:=a+\left(z^{*}\right)^{k}(b-a)$ for all $k \in \mathbb{N}$. It follows that $\operatorname{ker}\left(\tau_{*}\right)=\bigcup_{n \in \mathbb{N}} \operatorname{ker}\left(\Theta^{n} \tau\right)=$ $(0, \infty) \backslash\left\{b_{n}\right\}_{n=1}^{\infty}$. Thus, $\tau_{*}(x)=1_{\left\{b_{n}\right\}_{n=1}^{\infty}}(x)$ for all $x>0$. Since $\mathcal{L} \tau_{*}\left(b_{n}\right)=0$ for any $n$, we conclude the induced stopping time $\mathcal{L} \tau_{*}(x)=0$ for all $x$. Therefore this stopping law is essentially the same as the trivial equilibrium.

In the above example, when the probability distortion is convex or inverse S-shaped, the assumption (2) in Ebert and Strack (2017) is satisfied; hence the argument therein applies and equilibrium strategy is to stop immediately, or "never, ever getting started". However, when the distortion is concave which invalidates the assumption above, even a highly left-skewed gamble can be attractive due to the exaggeration of the small probabilities of extremely good events. As a result, all the selves can agree upon a threshold level before which they will all continue to hold the asset.

Example 5.2. Let $\beta>0$. Take $u(x)=(x-K)^{+}$for some $K>0$ and $w(x)=\eta x^{2}+(1-\eta) x$ for some $\eta \in(0,1)$. As shown in Example 4.2, time-inconsistency is present in this case.

For an arbitrary constant $b>\frac{\eta+1}{\eta} K$, define the stopping law $\tau \in \mathcal{T}\left(\mathbb{R}_{+}\right)$by

$$
\tau(x):=1_{(0, b)}(x), \quad \text { for all } x>0,
$$


which is different from the naïve one derived in Example 4.2. Observe that $\mathcal{L}^{*} \tau(x)=\inf \{t>$ $\left.0 \mid X_{t}^{x} \geq b\right\}$. For $x \geq b, \mathcal{L}^{*} \tau(x)=0$ and thus $J\left(x ; \mathcal{L}^{*} \tau(x)\right)=u(x)$, which implies $[b, \infty) \subseteq I_{\tau}$. For $0<x<b$, Lemma 5.1 (iii) gives

$$
J\left(x ; \mathcal{L}^{*} \tau(x)\right)=w\left(\frac{x}{b}\right) u(b)=\left(\eta \frac{x^{2}}{b^{2}}+(1-\eta) \frac{x}{b}\right)(b-K) .
$$

For $0<x \leq K, J\left(x ; \mathcal{L}^{*} \tau(x)\right)>0=u(x)$ and thus $(0, K] \subseteq C_{\tau}$. For $K<x<b$,

$$
\begin{aligned}
J\left(x ; \mathcal{L}^{*} \tau(x)\right)-u(x) & =\left(\eta \frac{x^{2}}{b^{2}}+(1-\eta) \frac{x}{b}\right)(b-K)-(x-K) \\
& =\frac{\eta(b-K)}{b^{2}}\left[x^{2}-\left(b+\frac{K b}{\eta(b-K)}\right) x+\frac{K b^{2}}{\eta(b-K)}\right] \\
& =\frac{\eta(b-K)}{b^{2}}(x-b)\left(x-b^{\prime}\right),
\end{aligned}
$$

where $b^{\prime}:=\frac{K b}{\eta(b-K)}>K$; moreover, $b>\frac{\eta+1}{\eta} K$ is equivalent to $\frac{K}{\eta(b-K)}<1$, and thus $b^{\prime}<b$. It follows that $J\left(x ; \mathcal{L}^{*} \tau(x)\right)-u(x)>0$ for $K<x<b^{\prime}, J\left(x ; \mathcal{L}^{*} \tau(x)\right)-u(x)<0$ for $b^{\prime}<x<b$, and $J\left(x ; \mathcal{L}^{*} \tau\left(b^{\prime}\right)\right)-u\left(b^{\prime}\right)=0$. We therefore conclude that $S_{\tau}=\left(b^{\prime}, b\right), C_{\tau}=\left(0, b^{\prime}\right)$, and $I_{\tau}=\left\{b^{\prime}\right\} \cup[b, \infty)$, which implies

$$
\tau_{1}:=\Theta \tau(x)=1_{\left(0, b^{\prime}\right]}(x) \text { for all } x>0 .
$$

By the same argument as above, we obtain $\left[b^{\prime}, \infty\right) \subseteq I_{\tau_{1}},(0, K] \subseteq C_{\tau_{1}}$, and when $K<x<b^{\prime}$,

$$
J\left(x ; \mathcal{L}^{*} \tau_{1}(x)\right)-u(x)=\frac{\eta\left(b^{\prime}-K\right)}{\left(b^{\prime}\right)^{2}}\left(x-b^{\prime}\right)\left(x-b^{\prime \prime}\right), \quad \text { with } \quad b^{\prime \prime}:=\frac{K b^{\prime}}{\eta\left(b^{\prime}-K\right)} .
$$

We claim that $b^{\prime \prime}>b^{\prime}$. First, observe that $b^{\prime}<\frac{\eta+1}{\eta} K$. Indeed, $b^{\prime} /\left(\frac{\eta+1}{\eta} K\right)=\frac{b}{(\eta+1)(b-k)}<1$ if and only if $0<\eta(b-K)-K$, which is equivalent to $b>\frac{\eta+1}{\eta} K$. Now, since $b^{\prime}<\frac{\eta+1}{\eta} K$ yields $\frac{K}{\eta\left(b^{\prime}-K\right)}>1$, we get $b^{\prime \prime}>b^{\prime}$. It follows that $J\left(x ; \mathcal{L}^{*} \tau_{1}(x)\right)-u(x)>0$ for all $K<x<b^{\prime}$. Now we conclude that $C_{\tau_{1}}=\left(0, b^{\prime}\right)$ and $I_{\tau_{1}}=\left[b^{\prime \prime}, \infty\right)$, which shows that

$$
\Theta \tau_{1}(x)=1_{\left(0, b^{\prime}\right]}(x)=\tau_{1}(x) \text { for all } x>0 .
$$

That is, $\tau_{*}(x)=\lim _{n \rightarrow \infty} \Theta^{n} \tau(x)=\Theta \tau(x)=1_{\left(0, b^{\prime}\right]}(x)$ for all $x>0$. So this equilibrium strategy stops earlier than the initial stopping law before a strategic reasoning has been carried out, showing a less risk-taking attitude 9

An interesting question is what the fixed-point iteration leads to if one starts with a naïve law. In the next section we will study in great detail those equilibria $\hat{\tau}_{*}$ obtained from iterations on initially naïve stopping strategies, i.e. $\hat{\tau}$ in (2.5).

\footnotetext{
${ }^{9}$ Once again, this example does not contradict the result of Ebert and Strack (2017), as $u$ here is not strictly increasing, an assumption imposed in Ebert and Strack (2017).
} 


\section{A Detailed Study on the Case $\beta>0$}

In this section, we study in more details the case $\beta>0$, which, as shown in Section 4 , is the only case where genuine time-inconsistency may arise. As in Xu and Zhou (2013), we will discuss various parameter specifications related to the shapes of $u$ and $w$. We are particularly interested in investigating whether there is time-inconsistency, and comparing the naïve stopping law $\hat{\tau}$ in (2.5) with the corresponding equilibrium stopping law $\hat{\tau}_{*}$ in (3.6). Recall that when $\beta>0, u$ is nondecreasing with $u(0)=0$.

The next result, which will be used repeatedly in this section, shows that a drastic change in behavior takes place when a naïve agent applies the game-theoretic reasoning described in Section 2 just once: a naïve agent who would have never stopped ("until the bitter end" Ebert and Strack (2015)) transforms himself to a sophisticated one who stops immediately.

Proposition 6.1. Let $\beta>0$ and assume $u(x)>0 \forall x>0$. Then for $\tau \in \mathcal{T}\left(\mathbb{R}_{+}\right)$given by $\tau(x)=1 \forall x>0$,

$$
\tau_{*}(x)=\lim _{n \rightarrow \infty} \Theta^{n} \tau(x)=\Theta \tau(x)=0 \quad \forall x>0 .
$$

Proof. First of all $\beta>0$ implies $u$ is nondecreasing and $u(0)=0$. By the definition of $\tau$, $\mathcal{L}^{*} \tau(x)=\infty \forall x>0$. This, together with (4.6), implies $J\left(x ; \mathcal{L}^{*} \tau\right)=\int_{0}^{\infty} w(\mathbb{P}[u(0)>y]) d y=$ $0<u(x) \forall x>0$. It follows that $S_{\tau}=\mathbb{R}_{+}$, whence $\Theta \tau(x)=0 \forall x>0$.

Example 6.1. We have shown in Example 4.1 that, as $\alpha>1$, a naïve agent postpones stopping indefinitely, i.e. $\hat{\tau}(x)=1$ for all $x>0$. By Proposition 6.1, the corresponding equilibrium stopping law $\hat{\tau}_{*}=\lim _{n \rightarrow \infty} \Theta^{n} \hat{\tau}=\Theta \hat{\tau}$ is the trivial equilibrium described in Remark 2.4, namely, to stop immediately.

The naïvité, once thinking like a sophisticate, decides to stop immediately. What constitutes the economic reasoning behind the proof of Proposition 6.1 is the following. At any given point of time, assuming all the future selves will be carrying out the naïve, neverstopping strategy. With this in mind, the question is what to do now. There are only two options. If he is to follow the original strategy, namely, to continue now, then according to (4.6) the value of the (un-stopped) underlying process will almost surely diminish to zero and hence the payoff is zero. If he is to stop now, then he can get some positive payoff because of the assumption $u(x)>0$ when $x>0$. This simple comparison will prompt him to stop immediately.

In what follows we categorize various specifications of the shapes of $u$ and $w$.

\subsection{Convex $u$}

As discussed in Remark 4.1, $u$ being nondecreasing and convex generally indicates that the asset is "average" in terms of its risk-return profile. Also recall from Section 4.2 of $\mathrm{Xu}$ and Zhou (2013) that, when $u$ is convex, problem (2.1), with $J(x ; \tau)$ specified in (4.9), can be reduced to (4.16). If an optimizer $\lambda^{*}(x) \in(0,1]$ exists, then $\hat{\tau}_{x}:=\inf \left\{t \geq 0: X_{t}^{x} \geq\right.$ $\left.x / \lambda^{*}(x)\right\}$ is a pre-committed optimal stopping time. If an optimizer of (4.16) does not exist, then such a pre-committed optimal stopping time does not exist. 
If $w$ is concave, then the agent is risk-seeking or optimistic: he exaggerates the probability of the "very good" scenarios, and understates the probability of the "very bad" scenarios. With only an average asset at hand, this optimistic view however prevents the agent from liquidating the asset, as we show now. Assume $u$ is a convex function which is not linear on the entire domain $[0, \infty)$, and $w$ is concave. The same argument in Corollary 4.3 in $\mathrm{Xu}$ and Zhou (2013) shows that $\sup _{\tau \in \mathcal{T}} J(x ; \tau)>u(x)$ for all $x>0$. Indeed,

$$
\begin{aligned}
\sup _{\tau \in \mathcal{T}} J(x ; \tau) & =\sup _{0<a \leq x \leq b}\left[u(a)+w\left(\frac{x-a}{b-a}\right)(u(b)-u(a))\right] \\
& \geq \sup _{0<a \leq x \leq b}\left[u(a)+\frac{x-a}{b-a}(u(b)-u(a))\right] \\
& =\sup _{0<a \leq x \leq b}\left[\frac{b-x}{b-a} u(a)+\frac{x-a}{b-a} u(b)\right]>u(x),
\end{aligned}
$$

where the second line follows from $w(z) \geq z$ for all $z \in(0,1)$ (as $w$ is concave with $w(0)=0$ and $w(1)=1$ ), and the last inequality stems from the fact that $u$ is convex, but not linear on the entire $[0, \infty)$. Consequently, it is optimal for the agent to continue at any state $x$. The naïve stopping law is therefore $\hat{\tau}(x)=1$ for all $x>0$. If in addition $u$ satisfies $u(x)>0$ $\forall x>0$, then the corresponding equilibrium is $\hat{\tau}_{*}(x)=\Theta \hat{\tau}(x)=0$ for all $x>0$, thanks to Proposition 6.1.

Here, as in Example 6.1, if an agent examines the never-stop strategy by strategically thinking about his future selves' moves then he is a sophisticate who will stop immediately (or indeed will not start at all).

If, on the other hand, $w$ is convex, then the agent is pessimistic: he understates the probability of a "very good" scenario, and exaggerates the probability of a "very bad" scenario. The following example illustrates the behavior of this type of agents with an average asset at hand.

Example 6.2. Take $u(x)=x^{\eta}$ and $w(x)=x^{\gamma}$ with $\eta, \gamma>1$. Then (4.16) becomes $\sup _{\lambda \in(0,1]} \lambda^{\gamma-\eta} x^{\eta}$. If $\gamma>\eta, \lambda^{*}=1$ is the maximizer, and thus the optimal stopping time $\hat{\tau}_{x}=\inf \left\{t \geq 0: X_{t}^{x} \geq x\right\}=0$. If $\gamma=\eta$, $\sup _{\lambda \in(0,1]} \lambda^{\gamma-\eta} x^{\eta}=x^{\eta}=u(x)$, and thus $\hat{\tau}_{x}=0$ is optimal. Hence there is no time-inconsistency when $\gamma \geq \eta$, and the optimal stopping law is to stop immediately.

The interesting case is when $\gamma<\eta$, in which case $\sup _{\lambda \in(0,1]} \lambda^{\gamma-\eta} x^{\eta}=\infty$, not attainable by any $\lambda \in(0,1]$. In this case, an optimal stopping time $\hat{\tau}_{x}$ fails to exist for all $x>0$. The naïve stopping law is therefore $\hat{\tau}(x)=1$ for all $x>0$. This leads to the equilibrium $\hat{\tau}_{*}(x)=\Theta \hat{\tau}(x)=0$ for all $x>0$, thanks to Proposition 6.1.

In the above example, note that $\gamma=w^{\prime}(1)$, which measures the intensity of probability weighting on very unfavorable events. So, when the agent sufficiently inflates the probabilities of bad scenarios relative to how good the asset is (i.e. $\gamma \geq \eta$ ), he liquidates the asset immediately no matter what type of agent he is. Otherwise (i.e. $\gamma<\eta$ ), he always intends to continue if he is naïve, and stops right away if he is sophisticated.

Also note in the above example that $w^{\prime}(0)=0$, indicating that the agent infinitely understates the probability of "very good" events. A slightly more hopeful agent, modeled 
by $w^{\prime}(0)>0$, who only moderately understates the probability of "very good" scenarios, may behave markedly different. This is shown in the next example.

Example 6.3. Take $u(x)=(x-K)^{+}$for some $K>0$ and $w(x)=\eta x^{2}+(1-\eta) x$ for some $\eta \in(0,1)$. We have studied this case in Examples 4.2 and 5.2. In particular, we have derived the naïve stopping law to be

$$
\hat{\tau}(x)=1_{\left(0, \frac{\eta+1}{\eta} K\right)}(x), \quad \text { for all } x>0 .
$$

Remarkably, this is also an equilibrium stopping law. To see this, for $x \geq \frac{\eta+1}{\eta} K, \mathcal{L}^{*} \hat{\tau}(x)=0$ and thus $J\left(x ; \mathcal{L}^{*} \hat{\tau}(x)\right)=u(x)$. This implies $\left[\frac{\eta+1}{\eta} K, \infty\right) \subset I_{\hat{\tau}}$. For $0<x<\frac{\eta+1}{\eta} K, \mathcal{L}^{*} \hat{\tau}(x)=$ $\inf \left\{t \geq 0: X_{t}^{x} \geq \frac{\eta+1}{\eta} K\right\}$. By Lemma 5.1\}(iii),

$$
J\left(x ; \mathcal{L}^{*} \hat{\tau}(x)\right)=w\left(\frac{\eta x}{(\eta+1) K}\right) u\left(\frac{\eta+1}{\eta} K\right)=\frac{\eta^{2} x^{2}}{(\eta+1)^{2} K}+\frac{1-\eta}{1+\eta} x .
$$

If $0<x \leq K, J\left(x ; \mathcal{L}^{*} \hat{\tau}(x)\right)>0=u(x)$, which yields $(0, K] \subset C_{\hat{\tau}}$. If $K<x<\frac{\eta+1}{\eta} K$,

$$
\begin{aligned}
J\left(x ; \mathcal{L}^{*} \hat{\tau}(x)\right)-u(x) & =\frac{\eta^{2} x^{2}}{(\eta+1)^{2} K}+\frac{1-\eta}{1+\eta} x-(x-K) \\
& =\frac{\eta^{2}}{(\eta+1)^{2} K}\left(x-\frac{\eta+1}{\eta} K\right)^{2}>0
\end{aligned}
$$

which shows that $\left(K, \frac{\eta+1}{\eta} K\right) \subset C_{\hat{\tau}}$. Hence, $C_{\hat{\tau}}=\left(0, \frac{\eta+1}{\eta} K\right)$ and $I_{\hat{\tau}}=\left[\frac{\eta+1}{\eta} K, \infty\right)$, which implies $\Theta \hat{\tau}(x)=1_{\left(0, \frac{\eta+1}{\eta} K\right)}(x)+\hat{\tau}(x) 1_{\left[\frac{\eta+1}{\eta} K, \infty\right)}(x)=\hat{\tau}(x)$ for all $x>0$, i.e. $\hat{\tau} \in \mathcal{E}\left(\mathbb{R}_{+}\right)$. We therefore conclude that $\hat{\tau}_{*}=\hat{\tau}$.

Examples 6.3 and 6.2 have the same qualitative properties for $u$ and $w$ - they are both convex. However, both types of agents display significantly different stopping behaviors. The reason behind is that $w^{\prime}(0)>0$ in Example 6.3 (instead of $w^{\prime}(0)=0$ in Example 6.2): the agent only moderately understates the probability of the "very good" scenarios. With this more hopeful view about the very good states, not only a naïve agent but also a sophisticated one is willing to exploit the upside potential of the average asset until its value hits a target, $x^{*}=\frac{\eta+1}{\eta} K$. Moreover, the two types of agent share the same "stop-gain" threshold in this particular example 10 Finally, a simple comparative static analysis shows that the more hopeful about the very good states of the world (i.e. the smaller $\eta$ ) the higher target he will set (i.e. the larger $x^{*}$ ), leading to a delayed stopping and more risk-seeking attitude.

Next, let us consider an $S$-shaped $w$, which indicates that the agent understates the probabilities of both the "very good" and "very bad" scenarios. Compared with a convex $w$, this reflects a less pessimistic view on the "very bad" scenarios, and is therefore more likely to lead the agent to postpone stopping. Indeed, Example 4.1 shows that an $S$-shaped $w$ (i.e. when $\alpha>1$ therein) induces time-inconsistency, making a naïve agent to postpone stopping indefinitely and a sophisticated agent to stop immediately.

\footnotetext{
${ }^{10}$ Recall that in Example 5.2 we have shown that the equilibrium law constructed via iterations on a non-naïve, threshold-type of stopping law is still of threshold type, yet with a smaller threshold.
} 
Finally, an inverse $S$-shaped $w$ indicates that the agent exaggerates the probabilities of both the "very good" and "very bad" scenarios. This type of $w$ has been widely studied, as it is consistent with empirical data11. The literature on inverse $S$-shaped $w$, including Tverskv and Kahneman (1992), Lattimore et al. (1992), Camerer and Ho (1994), Wu and Gonzalez (1996), Birnbaum and McIntosh (1996), and Preled (1998), among others, has suggested three main models of $w: 1)$ the one-factor model

$$
w(x)=\frac{x^{\gamma}}{\left(x^{\gamma}+(1-x)^{\gamma}\right)^{1 / \gamma}} \quad \text { with } \gamma^{*} \leq \gamma<1
$$

where $\gamma^{*} \approx 0.279$ is the minimal value of $\gamma$ such that $w$ is nondecreasing; 2) the two-factor model

$$
w(x)=\frac{\alpha x^{\gamma}}{\alpha x^{\gamma}+(1-x)^{\gamma}} \quad \text { with } \alpha>0,0<\gamma<1 ;
$$

and 3) the two-factor model (4.15) with $0<\alpha<1$ and $\gamma>0$.

Compared with a convex $w$, an inverse $S$-shaped $w$ reveals a more optimistic view on the "very good" scenarios, and is therefore more likely to lead the agent to postpone stopping. Indeed, in Example 4.1, when $w$ is inverse $S$-shaped (i.e. with $\alpha<1$ ), the optimal value is infinite for all $x>0$ :

$$
\sup _{\tau \in \mathcal{T}} J(x ; \tau)=\lim _{\lambda \downarrow 0} w(\lambda) u\left(\frac{x}{\lambda}\right)=\lim _{\lambda \downarrow 0} \frac{\exp \left(-\gamma(-\log (\lambda))^{\alpha}\right)}{\lambda^{\eta}} x^{\eta}=\lim _{y \rightarrow \infty} \frac{e^{-\gamma y^{\alpha}}}{e^{-\eta y}} x^{\eta}=\infty,
$$

where the last equality follows from $\alpha<1$. If an optimal stopping time $\hat{\tau}_{x}$ exists, we must have $\hat{\tau}_{x}>0$ as stopping immediately does not attain the optimal value $($ as $u(x)<\infty)$. The naïve stopping law is therefore to continue perpetually and the sophisticated one is to stop immediately.

\subsection{Convex $w$}

As mentioned above, a convex $w$ indicates a pessimistic view throughout: the agent deflates the probability of the very good scenarios, and inflates the probability of the very bad scenarios. We single out this case for further discussions because a pre-committed optimal stopping time $\hat{\tau}_{x}$, among generally non-Markovian stopping strategies, inherently has a simple structure of "cut loss or take profit" : when $w$ is convex, Theorem $4.2 \mathrm{in} \mathrm{Xu}$ and Zhou (2013) shows that

$$
\sup _{\tau \in \mathcal{T}} J(x ; \tau)=\sup _{0 \leq a \leq x \leq b<\infty} J\left(x ; \tau_{a b}\right),
$$

where $\tau_{a b}:=\inf \left\{t \geq 0: X_{t}^{x} \notin(a, b)\right\}$ if $a<b$, and $\tau_{a b}:=0$ if $a=b=x$.

First consider the case when $u$ is concave, which normally indicates that the asset is "bad" in terms of its risk-adjusted return; recall Remark 4.1. With such a bad asset at hand, the pessimistic view from a convex $w$ is expected to lead the agent to liquidate the asset immediately. As shown in Corollary 4.3 of $\mathrm{Xu}$ and Zhou (2013), when $u$ is concave and $w$ is convex, $\hat{\tau}_{x}=0$ is indeed an optimal stopping time. The problem is therefore time

\footnotetext{
${ }^{11}$ He and Zhou (2016) relate an inverse $S$-shaped $w$ with the emotion of hope (hope for the "very good" scenarios) and fear (fear of the "very bad" scenarios) in decision making.
} 
consistent, and all the three types of agents stop immediately. The moral of this result is that "never lay your hands on a bad stock if you are pessimistic".

The case when $u$ is convex has been studied in the previous subsection; see in particular Examples 6.2 and 4.2 .

Finally, when $u$ is $S$-shaped, it normally indicates that the asset is "average" to "bad" in terms of its risk-return profile; see Remark 4.1. This happens for example if the original utility function $U$ in (4.2) is of log-type $(U(x):=\ln (1+x))$ with $\beta>1$, or of CARA-type $\left(U(x):=1-e^{-\gamma x}\right.$ for $\left.\gamma>0\right)$ with $0<\beta<1$.

We investigate $S$-shaped $u$ in the next two examples, and find that 1 ) there may or may not be time-inconsistency, and 2) both the naïve and sophisticated agents may take the threshold-type of strategy.

Example 6.4. Take $u(x)=\left(x^{1 / \beta}-K\right)^{+}$for some $\beta>1$ and $K>0$, and $w(x)=x^{\alpha}$ for some $\alpha \geq 1$. First, we claim that for each $x>0$,

$$
\sup _{0 \leq a \leq x \leq b<\infty} J\left(x ; \tau_{a b}\right)=\sup _{0 \leq a \leq K^{\beta} \wedge x, K^{\beta} \vee x \leq b<\infty} J\left(x ; \tau_{a b}\right) .
$$

For $x \leq K^{\beta}$, observe that if $a \leq x \leq b \leq K^{\beta}$, then $J\left(x ; \tau_{a b}\right)=0$ as $u(a)=u(b)=0$. This already implies that (6.3) is true. For $x \leq K^{\beta}$, by the convexity of $w$ and the concavity of $u$ on $\left[K^{\beta}, \infty\right)$, the same argument in Corollary 4.3 of Xu and Zhou (2013) shows that

$$
\sup _{K^{\beta} \leq a \leq x \leq b<\infty} J\left(x ; \tau_{a b}\right) \leq u(x) .
$$

It follows that (6.3) holds. Now, for any $a \leq K^{\beta} \wedge x$ and $b \geq K^{\beta} \vee x$,

$$
f(a, b):=J\left(x ; \tau_{a b}\right)=u(a)+w\left(\frac{x-a}{b-a}\right)(u(b)-u(a))=\left(\frac{x-a}{b-a}\right)^{\alpha}\left(b^{1 / \beta}-K\right),
$$

where the second equality follows from Lemma 5.1 and the third equality is due to $u(a)=0$ for $a \leq K^{\beta}$. Note that

$$
\frac{\partial f(a, b)}{\partial a}=-\alpha\left(\frac{x-a}{b-a}\right)^{\alpha-1} \frac{b-x}{(b-a)^{2}}\left(b^{1 / \beta}-K\right)<0
$$

which implies that for any fixed $b \geq K^{\beta} \vee x, f(0, b) \geq f(a, b)$ for all $0 \leq a \leq x$. Then

$$
\frac{\partial f(0, b)}{\partial b}=\frac{x^{\alpha}}{b^{\alpha+1}}\left[(1 / \beta-\alpha) b^{1 / \beta}+\alpha K\right]
$$

showing that $\left(0, b^{*}\right)$ with $b^{*}:=\left(\frac{\alpha K}{\alpha-1 / \beta}\right)^{\beta}>K^{\beta}$ is the maximizer of (6.3). We thus conclude that

$$
\hat{\tau}_{x}:=\tau_{0 b^{*}}=\inf \left\{t \geq 0: X_{t}^{x} \geq\left(\frac{\alpha K}{\alpha-1 / \beta}\right)^{\beta}\right\}
$$

is an optimal stopping time of (2.1), with $J(x ; \tau)$ specified in (4.9), for each $x>0$. There in no time-inconsistency here as the stopping threshold $\left(\frac{\alpha K}{\alpha-1 / \beta}\right)^{\beta}$ is independent of current state $x>0$. In this case, all the three types of agents have the same (optimal) stopping law, which is

$$
\hat{\tau}(x)=1_{\left(0,\left(\frac{\alpha K}{\alpha-1 / \beta}\right)^{\beta}\right)}(x), \text { for all } x>0 .
$$


Example 6.5. Take $u(x)=\left(x^{1 / \beta}-K\right)^{+}$for some $\beta>1$ and $K>0$, and $w(x)=\frac{1}{2}\left(x^{2}+x\right)$. First, observe that (6.3) follows from the same argument in Example 6.4. For any $a \leq K^{\beta} \wedge x$ and $b \geq K^{\beta} \vee x$

$f(a, b):=J\left(x ; \tau_{a b}\right)=u(a)+w\left(\frac{x-a}{b-a}\right)(u(b)-u(a))=\frac{1}{2}\left[\left(\frac{x-a}{b-a}\right)^{2}+\frac{x-a}{b-a}\right]\left(b^{1 / \beta}-K\right)$,

where the second equality follows from Lemma 5.1 and the third equality is due to $u(a)=0$ for $a \leq K^{\beta}$. For any fixed $b \geq K^{\beta} \vee x$, observe that $f(0, b) \geq f(a, b)$ for all $0<a \leq x$. Then a direct calculation yields

$$
\frac{\partial f(0, b)}{\partial b}=\frac{x}{2 b^{3}} h(b), \quad \text { where } \quad h(b):=\left(\frac{1}{\beta}-1\right) b^{\frac{1}{\beta}+1}-\left(\frac{1}{\beta}-2\right) x b^{\frac{1}{\beta}}+K b+2 x K .
$$

We deduce from

$$
h^{\prime \prime}(b)=-\frac{1}{\beta}\left(1-\frac{1}{\beta}\right) b^{\frac{1}{\beta}-1}\left[\left(\frac{1}{\beta}+1\right)-\left(2-\frac{1}{\beta}\right) \frac{x}{b}\right]
$$

that on $(0, \infty), h$ starts being convex, becomes less convex as $b$ increases, and eventually turns concave. This, together with $h(0)=2 x K>0$ and $h(\infty)=-\infty$, shows that there exists a unique $b^{*}(x)>0$ such that $h\left(b^{*}\right)=0$. As a consequence, $h(x)>0$ if and only if $x<b^{*}(x)$. Since

$$
h(x)=x\left[\left(\frac{2}{\beta}-3\right) x^{\frac{1}{\beta}}+3 K\right]
$$

we conclude that $x<b^{*}(x)$ if and only if $x<\bar{x}:=\left(\frac{3 K}{3-2 / \beta}\right)^{\beta}$. Thus,

$$
\hat{\tau}_{x}:=\tau_{0 b^{*}}=\inf \left\{t \geq 0: X_{t}^{x} \geq b^{*}(x)\right\}= \begin{cases}>0 & \text { if } 0<x<\bar{x} \\ =0 & \text { if } x \geq \bar{x}\end{cases}
$$

is an optimal stopping time of (2.1), with $J(x ; \tau)$ specified in (4.9). Time-inconsistency is present here as the stopping threshold $b^{*}(x)$ depends on the current state $x$. The naïve stopping law is

$$
\hat{\tau}(x)=1_{(0, \bar{x})}(x), \quad \text { for all } x>0 .
$$

Remarkably, this is already an equilibrium stopping law. To see this, for $x \geq \bar{x}, \mathcal{L}^{*} \hat{\tau}(x)=0$ and thus $J\left(x ; \mathcal{L}^{*} \hat{\tau}(x)\right)=u(x)$. This shows that $[\bar{x}, \infty) \subseteq I_{\hat{\tau}}$. For $x<\bar{x}, \mathcal{L}^{*} \hat{\tau}(x)=\tau_{0 \bar{x}}=$ $\inf \left\{t \geq 0: X_{t}^{x} \geq \bar{x}\right\}$. Then

$$
J\left(x ; \mathcal{L}^{*} \hat{\tau}(x)\right)=w\left(\frac{x}{\bar{x}}\right) u(\bar{x})=\frac{1}{2}\left[\left(\frac{x}{\bar{x}}\right)^{2}+\frac{x}{\bar{x}}\right]\left(\bar{x}^{\frac{1}{\beta}}-K\right)^{+},
$$

where the first equality is due to Lemma 5.1. If $x \leq K^{\beta}, J\left(x ; \mathcal{L}^{*} \hat{\tau}(x)\right)>0=u(x)$, which implies $\left(0, K^{\beta}\right] \subseteq C_{\hat{\tau}}$. Now, observe that the curve

$$
y=g(x):=\frac{1}{2}\left[\left(\frac{x}{\bar{x}}\right)^{2}+\frac{x}{\bar{x}}\right]\left(\bar{x}^{\frac{1}{\beta}}-K\right)=\frac{u(\bar{x})}{2 \bar{x}^{2}}\left(x+\frac{\bar{x}}{2}\right)^{2}-\frac{u(\bar{x})}{8}
$$


is a convex quadratic function that intersects the concave function $y=\kappa(x):=x^{1 / \beta}-K$ at $x=\bar{x}$. Moreover, it can be checked that $g^{\prime}(\bar{x})=\kappa^{\prime}(\bar{x})$, which implies that $x=\bar{x}$ is the only intersection of these curves and $g(x)>\kappa(x)$ on $(0, \bar{x})$. If $x \in\left(K^{\beta}, \bar{x}\right)$, observe that $J\left(x ; \mathcal{L}^{*} \hat{\tau}(x)\right)=g(x)>\kappa(x)=u(x)$, which shows that $\left(K^{\beta}, \bar{x}\right) \subseteq C_{\hat{\tau}}$. We therefore conclude that $C_{\hat{\tau}}=(0, \bar{x})$ and $I_{\hat{\tau}}=[\bar{x}, \infty)$. It follows that $\Theta \hat{\tau}(x)=1_{(0, \bar{x})}+\hat{\tau}(x) 1_{[\bar{x}, \infty)}=\hat{\tau}(x)$ for all $x>0$. That is, $\hat{\tau} \in \mathcal{E}\left(\mathbb{R}_{+}\right)$, and thus $\hat{\tau}_{*}=\hat{\tau}$.

\subsection{Concave $u$}

When $u$ is concave, it normally indicates that the asset is "bad" in terms of its risk-return profile; recall Remark 4.1.

For a general $w$, to find an optimal stopping time $\hat{\tau}_{x}$ for (2.1), $\mathrm{Xu}$ and Zhou (2013) turn to the quantile formulation

$$
\sup _{G \in \mathcal{Q}} J_{Q}(x ; G)
$$

as stated in (4.13). Once the optimal quantile function $G_{x}^{*}$ is found, a pre-committed optimal stopping time $\hat{\tau}_{x}$ can be constructed using Skorokhod's embedding theorem such that the quantile function of $X_{\hat{\tau}_{x}}^{x}$ coincides with $G_{x}^{*}$; see $\mathrm{Xu}$ and Zhou (2013) for details.

When $w$ is convex (including the case of no probability distortion), the case has been covered in Subsection 6.2. When $w$ is also concave, the next result, taken from Theorem 5.1 of $\mathrm{Xu}$ and Zhou (2013), states in detail how to find the quantile function $G_{x}^{*}$ that maximizes (6.9).

Proposition 6.2. Suppose $u$ and $w$ are both concave. For each $x>0$, if there exists $\lambda^{*}(x) \geq 0$ such that

$$
\left(u^{\prime}\right)_{\ell}^{-1}\left(\frac{\lambda^{*}}{w^{\prime}(1-y)}\right)>0 \quad \forall y \in(0,1) \quad \text { and } \quad \int_{0}^{1}\left(u^{\prime}\right)_{\ell}^{-1}\left(\frac{\lambda^{*}}{w^{\prime}(1-y)}\right) d y=x
$$

where

$$
\left(u^{\prime}\right)_{\ell}^{-1}(y):=\inf \left\{z \geq 0: u^{\prime}(z) \leq y\right\}
$$

then $G_{x}^{*}(y):=\left(u^{\prime}\right)_{\ell}^{-1}\left(\frac{\lambda^{*}}{w^{\prime}(1-y)}\right)$ is an optimal solution to (6.9).

Intriguingly, in order to identify the naïve stopping law $\hat{\tau}$ it is not necessary to find out what $\hat{\tau}_{x}$ is specifically in this case: in view of Remark 4.2, $\hat{\tau}$ is well-defined once the optimal quantile function $G_{x}^{*}$ is known; see the last equality of (4.14). This leads to the following result.

Theorem 6.1. Suppose $u$ is strictly concave and continuously differentiable, and $w$ is concave with $w(y) \neq y$ for some $y \in(0,1)$. If an optimal quantile $G_{x}^{*}$ as specified in Proposition 6.2 exists for all $x>0$, then

$$
\begin{gathered}
\hat{\tau}(x)=1 \quad \text { for all } x>0, \\
\hat{\tau}_{*}(x)=0 \quad \text { for all } x>0,
\end{gathered}
$$

where $\hat{\tau} \in \mathcal{T}\left(\mathbb{R}_{+}\right)$is the naïve stopping law defined in (2.5), and $\hat{\tau}_{*} \in \mathcal{E}\left(\mathbb{R}_{+}\right)$is the corresponding equilibrium law defined in (3.6). 
Proof. Since $u$ is nondecreasing and strictly concave, $u^{\prime}(x-) \geq u^{\prime}(x+)>0$ for all $x>0$.

For each $x>0$, to prove $\hat{\tau}(x)=1$, it suffices to show that $G_{x}^{*}(\cdot) \not \equiv x$, in view of (4.14). If $\lambda^{*}(x)=0$, then $G_{x}^{*}(y)=\left(u^{\prime}\right)_{\ell}^{-1}(0)=\infty \neq x$. Assume $\lambda^{*}(x)>0$. Note that $\inf _{y \geq(0,1)} w^{\prime}(y)$ must be strictly less than $\sup _{y \in(0,1)} w^{\prime}(y)$, otherwise $w(y)=y$ for all $y \in(0,1)$. Thus, we can take $y_{1}, y_{2} \in(0,1), y_{1} \neq y_{2}$ such that $w^{\prime}\left(1-y_{1}\right) \neq w^{\prime}\left(1-y_{2}\right)$. Without loss of generality, assume $\eta_{1}:=\frac{\lambda^{*}}{w^{\prime}\left(1-y_{1}\right)}>\frac{\lambda^{*}}{w^{\prime}\left(1-y_{1}\right)}=: \eta_{2}$. Since $y \mapsto u^{\prime}(y)$ is continuous and strictly decreasing, $\left(u^{\prime}\right)_{\ell}^{-1}\left(\eta_{1}\right)$ must be strictly less than $\left(u^{\prime}\right)_{\ell}^{-1}\left(\eta_{2}\right)$, unless they are both $+\infty$. This already shows that $G_{x}^{*}(\cdot)$ cannot be a constant function equal to $x$.

With $\hat{\tau}(x)=1$ for all $x>0$, Proposition 6.1 implies $\hat{\tau}_{*}(x)=\Theta \hat{\tau}(x)=0$ for all $x>0$.

Example 6.6. Take $u(x)=\frac{1}{\gamma} x^{\gamma}$ for some $0<\gamma<1$, and $w(x)=x^{\alpha}$ for some $0<\alpha \leq 1$. Detailed analysis was carried out in Example 1 of $\mathrm{Xu}$ and Zhou (2013), where pre-committed optimal stopping times of (2.1), with $J(x ; \tau)$ specified in (4.9), were found, under different values of $\gamma$ and $\alpha$ : If $\alpha=1$ (i.e. no probability distortion), $\hat{\tau}_{x}:=0$ solves (2.1) and the problem is time consistent, as also argued at the start of Subsection 6.2. If $\gamma<\alpha<1$, i.e. the agent is moderately risk-seeking, then

$$
\hat{\tau}_{x}:=\inf \left\{t \geq 0: X_{t}^{x} \leq \frac{\alpha-\gamma}{1-\gamma} \max _{0 \leq s \leq t} X_{s}^{x}\right\}
$$

solves (2.1). If $\alpha<\gamma$, i.e. the agent is sufficiently risk-seeking, then for any $0<\eta<1$,

$$
\hat{\tau}_{x}:=\inf \left\{t \geq 0: X_{t}^{x} \leq \eta \max _{0 \leq s \leq t} X_{s}^{x}\right\}
$$

solves (2.1). In both latter cases, the pre-committed optimal strategies are non-Markovian, and are of the so-called "trailing stop" type in the context of selling a stock: the investor sells only when the stock price drops from the historical high (the "maximum drawdown") by a given percentage.

Since $\frac{\alpha-\gamma}{1-\gamma}<1$ and $\eta<1$, it follows from (6.11) and (6.12) that $\hat{\tau}_{x}>0$ for all $x>0$. Thus, the naïve agent will never stop as he reoptimizes at every time instant, i.e. $\hat{\tau}(x)=1$ for all $x>0$. It then follows from Proposition 6.1 that a sophisticated agent will stop immediately, i.e. $\hat{\tau}_{*}(x)=\Theta \hat{\tau}(x)=0$ for $x>0$. All these affirm Theorem 6.1,

Finally, let us consider the case when $w$ is inverse $S$-shaped. The next result, taken from Theorem 5.2 of $\mathrm{Xu}$ and Zhou (2013), states in detail how to find the optimal quantile function $G_{x}^{*}$.

Proposition 6.3. Suppose $u$ is concave and $w$ is inverse $S$-shaped, i.e. it is concave on $[0,1-q]$ and convex on $[1-q, 1]$ for some $q \in(0,1)$. For each $x>0$, if $\left(a^{*}(x), \lambda^{*}(x)\right)$ is a solution to the following mathematical program:

$$
\begin{array}{ll}
\text { Maximize } & (1-w(1-q)) u(a)+\int_{q}^{1} u\left(a \vee\left(u^{\prime}\right)_{\ell}^{-1}\left(\frac{\lambda}{w^{\prime}(1-y)}\right)\right) w^{\prime}(1-y) d y \\
\text { subject to } \quad a, \lambda \geq 0, \quad a q+\int_{q}^{1} a \vee\left(u^{\prime}\right)_{\ell}^{-1}\left(\frac{\lambda}{w^{\prime}(1-y)}\right) d y=x,
\end{array}
$$


where $\left(u^{\prime}\right)_{\ell}$ is defined as in (6.10), then

$$
G^{*}(y):=a^{*} 1_{(0, q]}(y)+\left(a^{*} \vee\left(u^{\prime}\right)_{\ell}^{-1}\left(\frac{\lambda^{*}}{w^{\prime}(1-y)}\right)\right) 1_{(q, 1)}(y), \quad y \in[0,1),
$$

is an optimal solution to (6.9).

When $w^{\prime}(0+)=\infty$, i.e. the agent infinitely exaggerates the probability of the "very good" scenarios, we have the following result.

Theorem 6.2. Suppose $u$ is concave and $w$ is inverse $S$-shaped with $w^{\prime}(0+)=\infty$. Assume that an optimal quantile $G_{x}^{*}$ as specified in Proposition 6.3 exists for all $x>0$.

(i) If $\sup _{y>0} u(y)$ is attained, then

$$
\hat{\tau}(x)=\hat{\tau}_{*}(x)=1_{\left(0, y^{*}\right)}(x) \text { for all } x>0 .
$$

where $y^{*}:=\inf \left\{y>0: u(y)=\sup _{z>0} u(z)\right\}<\infty$.

(ii) If $\sup _{y>0} u(y)$ is not attained, then

$$
\begin{gathered}
\hat{\tau}(x)=1 \quad \text { for all } x>0 \\
\hat{\tau}_{*}(x)=0
\end{gathered}
$$

Here, $\hat{\tau} \in \mathcal{T}\left(\mathbb{R}_{+}\right)$is the naïve stopping law defined in (2.5), and $\hat{\tau}_{*} \in \mathcal{E}\left(\mathbb{R}_{+}\right)$is the corresponding equilibrium law defined in (3.6).

Proof. Thanks to (4.14), for each $x>0, \hat{\tau}(x)=0$ if and only if $G_{x}^{*}(\cdot) \equiv x$.

(i) With $y^{*}<\infty$, we observe from $w^{\prime}(0+)=\infty$ that for any $\lambda \geq 0,\left(u^{\prime}\right)_{\ell}^{-1}\left(\frac{\lambda}{w^{\prime}(1-y)}\right) \uparrow y^{*}$ as $y \uparrow 1$. Then, for any $x>0$, (6.14) implies that $G_{x}^{*}(\cdot) \equiv x$ holds only when $a^{*}=x \geq y^{*}$. This already shows that $\hat{\tau}(x)=1$ for all $x<y^{*}$. Now, for $x \geq y^{*}$, observe that with $a=x$, the constraint in (6.13) becomes

$$
x q+\int_{q}^{1} x \vee\left(u^{\prime}\right)_{\ell}^{-1}\left(\frac{\lambda}{w^{\prime}(1-y)}\right) d y=x q+\int_{q}^{1} x d y=x .
$$

That is, the constraint is satisfied for any $\lambda \geq 0$. Similarly, with $a=x$ and any $\lambda \geq 0$, the objective function in (6.13) has the value

$$
(1-w(1-q)) u(x)+\int_{q}^{1} u(x) w^{\prime}(1-y) d y=u(x)=\sup _{y>0} u(y) .
$$

This already attains the maximum of (6.13). Indeed, the maximization in (6.13) can be simplified as

$$
\begin{aligned}
& \max _{a, \lambda \geq 0}\left\{(1-w(1-q)) u(a)+\int_{q}^{1} u\left(a \vee\left(u^{\prime}\right)_{\ell}^{-1}\left(\frac{\lambda}{w^{\prime}(1-y)}\right)\right) w^{\prime}(1-y) d y\right\} \\
= & \max _{a \geq y^{*}}\left\{(1-w(1-q)) u(a)+\int_{q}^{1} u(a) w^{\prime}(1-y) d y\right\}=\max _{a \geq y^{*}} u(a)=\sup _{y>0} u(y) .
\end{aligned}
$$


We therefore conclude that $a^{*}=x$ and any $\lambda \geq 0$ form a solution to (6.13), which yields $G_{x}^{*}(\cdot) \equiv x$. Thus, $\hat{\tau}(x)=0$ for all $x \geq y^{*}$. We therefore obtain $\hat{\tau}(x)=1_{\left(0, y^{*}\right)}(x)$ for all $x>0$. Finally, for any $x \geq y^{*}, \mathcal{L}^{*} \hat{\tau}(x)=0$ and thus $J\left(x ; \mathcal{L}^{*} \hat{\tau}(x)\right)=u(x)$, which implies $\Theta \hat{\tau}(x)=\hat{\tau}(x)=0$. For any $x<y^{*}, \mathcal{L}^{*} \hat{\tau}(x)$ is the first hitting time of $X^{x}$ to the value $y^{*}$. Thus, $J\left(x ; \mathcal{L}^{*} \hat{\tau}(x)\right)=\int_{0}^{\infty} w\left(\mathbb{P}\left[u\left(y^{*}\right)>y\right]\right) d y=u\left(y^{*}\right) \geq u(x)$. It follows that $\Theta \hat{\tau}(x)=1=\hat{\tau}(x)$. As a result, $\hat{\tau}_{*}(x)=\Theta \hat{\tau}(x)=\hat{\tau}(x)$ for all $x>0$.

(ii) If $\lambda^{*}=0$, then $\left(u^{\prime}\right)_{\ell}^{-1}\left(\frac{\lambda^{*}}{w^{\prime}(1-y)}\right)=\left(u^{\prime}\right)_{\ell}^{-1}(0)=\infty$ for all $y \in(q, 1)$, as $\sup _{y>0} u(y)$ is not attained. This implies that the constraint in (6.13) cannot be satisfied, a contradiction. Assume $\lambda^{*}>0$. Since $w^{\prime}(0+)=\infty, \frac{\lambda^{*}}{w^{\prime}(1-y)} \rightarrow 0$ as $y \uparrow 1$. The concavity of $u$ and the fact that $\sup _{y>0} u(y)$ is not attained then imply that $\left(u^{\prime}\right)_{\ell}^{-1}\left(\frac{\lambda^{*}}{w^{\prime}(1-y)}\right) \rightarrow \infty$ as $y \uparrow 1$, which shows $G_{x}^{*}(\cdot) \not \equiv x$. Thus, $\hat{\tau}(x)=1$ for all $x>0$. By Proposition 6.1 , $\hat{\tau}_{*}(x)=0$ for all $x>0$.

Since all the three major forms of $w$ proposed in the literature and supported by empirical evidence, (6.1), (6.2), and (4.15) with $0<\alpha<1$ and $\gamma>0$, are inverse $S$-shaped with $w^{\prime}(0+)=\infty$, the result of Theorem 6.2 is of sufficient practical relevance.

Theorem 6.2-(ii) is consistent with the results of Ebert and Strack (2015) and Ebert and Strack (2017): the naïve agent never stops while the sophisticated one stops immediately. Both Ebert and Strack (2015) and Ebert and Strack (2017) assume that $u$ is strictly increasing, which is stronger than the condition that $\sup _{y>0} u(y)$ is not attained. On the other hand, Theorem 6.2 (i) shows that if there exists a state that maximizes the payoff function $u$ itself, then it makes no sense for even the naïvité to hold the asset forever: he ought to stop whenever such a state is reached. Moreover, since such a threshold type strategy can be upheld by all the selves, it is also a sophisticated strategy. Note that this conclusion also demonstrates that the respective extreme stopping behaviors of the two types of agents reported in Ebert and Strack (2015) and Ebert and Strack (2017) depend critically on the assumption that the payoff function $u$ is strictly increasing. When $\sup _{y>0} u(y)$ is attained which violates the assumption, then both agents will instead adopt the same, threshold-type of strategy.

Even when an inverse $S$-shaped $w$ admits $w^{\prime}(0+)<\infty$, meaning that the agent only modestly inflates the probabilities of very good states of nature, a naïve agent may still postpone stopping indefinitely, as the next example shows. This example was first studied in Subsection 5.2 of $\mathrm{Xu}$ and Zhou (2013), where the authors demonstrated how to find a precommitted optimal stopping time, but did not solve it in a completely explicit fashion. In the following, we will solve the problem completely and explicitly, correct a typo in calculation on p.270 of $\mathrm{Xu}$ and Zhou (2013), and argue that a naïve agent will never stops.

Example 6.7. Consider $u(x)=\frac{1}{\gamma} x^{\gamma}$ with $0<\gamma<1$, and an inverse $S$-shaped probability weighting function

$$
w(x):= \begin{cases}2 x-2 x^{2}, & \text { if } 0 \leq x \leq 1 / 2, \\ 2 x^{2}-2 x+1, & \text { if } 1 / 2 \leq x \leq 1 .\end{cases}
$$

Using the mathematical program in Proposition 6.3, $\mathrm{Xu}$ and Zhou (2013) shows that the problem (6.9) reduces to

$$
\sup _{c \in\left(\frac{1}{2}, 1\right]} \frac{1}{\gamma} x^{\gamma} g(c)
$$


where

$g(c):=\left(\frac{1}{c+\frac{1-\gamma}{2(2-\gamma)}\left[(2 c-1)^{\frac{1}{\gamma-1}}-(2 c-1)\right]}\right)^{\gamma}\left(1-2 c+2 c^{2}+\frac{1-\gamma}{2-\gamma}\left[(2 c-1)^{\frac{\gamma}{\gamma-1}}-(2 c-1)^{2}\right]\right)$,

and the optimal quantile function is given by

$$
G_{x}^{*}(y)=a(x) 1_{\left(0, c^{*}\right]}(y)+a(x)\left(\frac{4 c^{*}-2}{4 y-2}\right)^{1 /(\gamma-1)} 1_{\left(c^{*}, 1\right)}(y), \quad y \in[0,1),
$$

where $c^{*}$ is the maximizer of (6.15) and

$$
a(x):=\frac{x}{c^{*}+\frac{1-\gamma}{2(2-\gamma)}\left[\left(2 c^{*}-1\right)^{\frac{1}{\gamma-1}}-\left(2 c^{*}-1\right)\right]} .
$$

The corresponding Azéma-Yor stopping time

$$
\hat{\tau}_{x}:=\inf \left\{t \geq 0: \Psi\left(X_{t}^{x}\right) \leq \max _{0 \leq s \leq t} X_{s}^{x}\right\}
$$

is optimal for problem (2.1), with $J(x ; \tau)$ specified in (4.9), where $\Psi$ is the barycenter function for $F^{*}$ defined by

$$
\begin{aligned}
\Psi(z) & := \begin{cases}0, & \text { if } z<a(x), \\
\left(\int_{z}^{\infty} y d F_{x}^{*}(y)\right) /\left(1-F_{x}^{*}(z-)\right), & \text { if } a(x) \leq z<\left(2 c^{*}-1\right)^{\frac{1}{\gamma-1}} a(x), \\
z, & \text { if } z \geq\left(2 c^{*}-1\right)^{\frac{1}{\gamma-1}} a(x),\end{cases} \\
& = \begin{cases}0, & \text { if } z<a(x), \\
\frac{1-\gamma}{2-\gamma} \frac{\left(2 c^{*}-1\right)^{\frac{2-\gamma}{\gamma-1}}-(z / a(x))^{2-\gamma}}{\left(2 c^{*}-1\right)^{-1}-(z / a(x))^{1-\gamma}} a(x), & \text { if } a(x) \leq z<\left(2 c^{*}-1\right)^{\frac{1}{\gamma-1}} a(x), \\
z, & \text { if } z \geq\left(2 c^{*}-1\right)^{\frac{1}{\gamma-1}} a(x) .\end{cases}
\end{aligned}
$$

Note that (6.18) corrects the formula of $\Psi$ on p.270 of Xu and Zhou (2013).

The above procedure hinges on the solvability of the maximizer $c^{*} \in(1 / 2,1]$. Xu and Zhou (2013) did not solve for $c^{*}$, but only mentioned that the maximizer exists if $0<\gamma<2 / 3$. Here, we claim that $c^{*}$ is actually a constant $1 / \sqrt{2}$, independent of the choice of $0<\gamma<1$. To see this, observe that

$$
g^{\prime}(c)=\frac{\gamma}{(2 c-1)(2-\gamma)}\left(\frac{2(2-\gamma)}{2 c+(1-\gamma)\left[1+(2 c-1)^{\frac{1}{\gamma-1}}\right]}\right)^{1+\gamma} h(c),
$$

where

$$
\begin{aligned}
h(c) & :=1+(2 c-1)^{\frac{1}{\gamma-1}}-2 c(2 c-1)^{\frac{\gamma}{\gamma-1}}-2 c(1-c)\left[1+2 c+(2 c-1)^{\frac{1}{\gamma-1}}\right] \\
& =(1-2 c(1-c)(1+2 c))+\left[(1-2 c(1-c))(2 c-1)^{\frac{1}{\gamma-1}}-2 c(2 c-1)^{\frac{\gamma}{\gamma-1}}\right] \\
& =\left(1-2 c-2 c^{2}+4 c^{2}\right)+(2 c-1)^{\frac{1}{\gamma-1}}\left(1-2 c+2 c^{2}-2 c(2 c-1)\right) \\
& =\left(2 c^{2}-1\right)(2 c-1)-(2 c-1)^{\frac{1}{\gamma-1}}\left(2 c^{2}-1\right) \\
& =\left(2 c^{2}-1\right)\left[(2 c-1)-(2 c-1)^{\frac{1}{1-\gamma}}\right] .
\end{aligned}
$$


Note that the only real solutions to $h(c)=0, c \in(1 / 2,1]$, are $c=1 / \sqrt{2}$ and $c=1$. Since $h(c) \uparrow \infty$ as $c \downarrow 1 / 2$, we must have $h>0$ on $(1 / 2,1 / \sqrt{2})$ and $h<0$ on $(1 / \sqrt{2}, 1)$. In view of (6.19), this already implies that the maximizer of (6.15) is $c^{*}=1 / \sqrt{2}$, for any given $0<\gamma<1$.

At any current state $x>0$, to decide whether to stop or not under the optimal stopping time $\hat{\tau}_{x}$ in (6.17), it boils down to determine if $\Psi(x) \leq x$ holds. With $c^{*}=1 / \sqrt{2}$, we observe from (6.16) that $a(x)$ is decreasing in $\gamma$, and $a(x) \uparrow 2(\sqrt{2}-1) x<x$ as $\gamma \downarrow 0$. This implies $a(x)<x$ for all $0<\gamma<1$. Similarly, $\left(2 c^{*}-1\right)^{\frac{1}{\gamma-1}} a(x)$ is increasing in $\gamma$, and $\left(2 c^{*}-1\right)^{\frac{1}{\gamma-1}} a(x) \downarrow 2 x$ as $\gamma \downarrow 0$. This implies that $\left(2 c^{*}-1\right)^{\frac{1}{\gamma-1}} a(x)>x$ for all $0<\gamma<1$. Thus, given any $0<\gamma<1$, we have $a(x)<x<\left(2 c^{*}-1\right)^{\frac{1}{\gamma-1}} a(x)$ for all $x>0$. In view of (6.18), this implies

$$
\Psi(x)=\frac{1-\gamma}{2-\gamma} \frac{\left(2 c^{*}-1\right)^{\frac{2-\gamma}{\gamma-1}}-(x / a(x))^{2-\gamma}}{\left(2 c^{*}-1\right)^{-1}-(x / a(x))^{1-\gamma}} a(x) .
$$

Using (6.16) and $c^{*}=1 / \sqrt{2}$, it can be checked that $\Psi$ is increasing in $\gamma$, and $\Psi(x) \downarrow(3 / 2) x$ as $\gamma \downarrow 0$. Thus, for any $0<\gamma<1, \Psi(x)>x$ and thus $\hat{\tau}_{x}>0$ for all $x>0$. It follows that the naïve stopping law is $\hat{\tau}(x)=1$ for all $x>0$, and by Proposition 6.1 the equilibrium stopping law is $\hat{\tau}_{*}(x)=\Theta \hat{\tau}(x)=0$ for all $x>0$.

\subsection{S-shaped $u$}

When $u$ is $S$-shaped, it normally indicates that the asset is "average" to "bad" in terms of its risk-return profile. As in Xu and Zhou (2013), Section 6, we discuss only the case where $w$ is inverse $S$-shaped, as the other cases either have been treated or can be treated similarly as in the previous subsections.

Suppose $u$ is convex on $[0, \theta]$ and concave on $[\theta, \infty)$ for some $\theta>0$, and $w$ is concave on $[0,1-q]$ and convex on $[1-q, 1]$ for some $q \in(0,1)$. One may solve the problem (6.9) using similar arguments in Propositions 6.2 and 6.3. More specifically, Section 6 of $\mathrm{Xu}$ and Zhou (2013) proposes the following mathematical program: if there exist $0<a_{1} \leq a_{2} \leq a_{3} \leq \theta$, $0 \leq c_{1} \leq c_{2} \leq q$, and $\lambda \geq 0$ such that they maximize

$$
\begin{aligned}
\left(1-w\left(1-c_{1}\right)\right) u\left(a_{1}\right) & +\left(w\left(1-c_{1}\right)-w\left(1-c_{2}\right)\right) u\left(a_{2}\right)+\left(w\left(1-c_{2}\right)-w(1-q)\right) u\left(a_{3}\right) \\
& +\int_{q}^{1} u\left(a_{3} \vee\left(u^{\prime}\right)_{\ell}^{-1}\left(\frac{\lambda}{w^{\prime}(1-y)}\right)\right) w^{\prime}(1-y) d y
\end{aligned}
$$

subject to

$$
a_{1} c_{1}+a_{2}\left(c_{2}-c_{1}\right)+a_{3}\left(q-c_{2}\right)+\int_{q}^{1} a_{3} \vee\left(u^{\prime}\right)_{\ell}^{-1}\left(\frac{\lambda}{w^{\prime}(1-y)}\right) d y \leq x,
$$

then

$$
\begin{aligned}
G_{x}^{*}(y):= & a_{1} 1_{\left(0, c_{1}\right]}(y)+a_{2} 1_{\left(c_{1}, c_{2}\right]}(y)+a_{3} 1_{\left(c_{2}, q\right]}(y) \\
& +\left(a_{3} \vee\left(u^{\prime}\right)_{\ell}^{-1}\left(\frac{\lambda}{w^{\prime}(1-y)}\right)\right) 1_{(q, 1)}(y), \quad y \in[0,1),
\end{aligned}
$$


is an optimal quantile function.

Here, we observe that under the general condition on $w$ specified in Theorem 6.2, the same argument there shows that $G_{x}^{*}$ in (6.20) is not a constant function equal to $x$. Hence, by (4.14), the naïve stopping law is $\hat{\tau}(x)=1$ for all $x>0$, and the corresponding equilibrium law is $\hat{\tau}_{*}(x)=\Theta \hat{\tau}(x)=0$ for all $x>0$.

\section{A Proof of Lemma 3.1}

First, recall from Problem 7.18 on p. 94 of Karatzas and Shreve (1991) that if $W$ is a standard Brownian motion defined on a probability space $\left(\Omega^{\prime}, \mathcal{F}^{\prime}, \mathbb{P}^{\prime}\right)$, then

$$
\mathbb{P}^{\prime}\left[\bar{W}_{t}>0\right]=\mathbb{P}^{\prime}\left[\underline{W}_{t}<0\right]=1, \quad \text { for all } t>0,
$$

where $\bar{W}_{t}:=\max _{s \in[0, t]} W_{s}$ and $\underline{W}_{t}:=\min _{s \in[0, t]} W_{s}$.

Fix $T>0$. With $Z$ in (3.2) being a martingale, we can define a probability $\mathbb{Q} \approx \mathbb{P}$ by $\frac{d \mathbb{Q}}{d \mathbb{P}}=Z_{T}$. Note that we have $\mathbb{Q} \approx \mathbb{P}$, instead of merely $\mathbb{Q} \ll \mathbb{P}$, because $Z_{T}>0 \mathbb{P}$-a.s. under (3.1). Girsanov's theorem then implies that under $\mathbb{Q}, d X_{t}=a\left(X_{t}\right) d \widetilde{B}_{t}$ for $t \in[0, T]$, where $\widetilde{B}_{t}:=B(t)+\int_{0}^{t} \theta\left(X_{s}\right) d s, t \in[0, T]$, is a $\mathbb{Q}$-Brownian motion. Since $X$ is a continuous local martingale under $\mathbb{Q}$, it can be expressed as a time-changed Brownian motion, i.e.

$$
X_{t}=x+W_{[X]_{t}} \quad t \in[0, T],
$$

for some standard Brownian motion $\left\{W_{t}\right\}_{t \geq 0}$ under $\mathbb{Q}$. As a consequence,

$$
\begin{aligned}
& \mathbb{Q}\left[\bar{X}_{T} \geq x+\eta\right]=\mathbb{Q}\left[X_{s} \geq x+\eta, \quad \text { for some } 0 \leq s \leq T\right] \\
& =\mathbb{Q}\left[W_{[X]_{s}} \geq \eta, \quad \text { for some } 0 \leq s \leq T\right] \\
& =\mathbb{Q}\left[W_{s} \geq \eta, \quad \text { for some } 0 \leq s \leq[X]_{T}\right] .
\end{aligned}
$$

This implies

$$
\left.\mathbb{Q}\left[\bar{X}_{T}^{x}>x\right]=\lim _{\eta \downarrow 0} \mathbb{Q}\left[\bar{X}_{T}^{x} \geq x+\eta\right]=\lim _{\eta \downarrow 0} \mathbb{E}^{\mathbb{Q}}\left[1_{\left\{W_{s} \geq \eta,\right.} \text { for some } 0 \leq s \leq[X]_{T}\right\}\right] .
$$

In view of (A.1), we have $\mathbb{Q}\left[\bar{W}_{t}>0\right]=1$ for all $t>0$. This implies that we can find some $\Omega^{*} \in \mathcal{F}$ with $\mathbb{Q}\left(\Omega^{*}\right)=1$ such that for each $\omega \in \Omega^{*}$, there exist a real sequence $\left\{t_{n}(\omega)\right\}$ with $t_{n}(\omega) \downarrow 0$ and $W_{t_{n}(\omega)}(\omega)>0$. It follows that for each $\omega \in \Omega^{*}$,

$$
\left.1_{\left\{W_{s} \geq \eta\right.} \text {, for some } 0 \leq s \leq[X]_{T}\right\}(\omega)=1 \text {, as } \eta \text { small enough. }
$$

We hence conclude from (A.2) that $\mathbb{Q}\left[\bar{X}_{T}^{x}>x\right]=1$. Similarly, we have

$$
\mathbb{Q}\left[\underline{X}_{T}^{x}<x\right]=\lim _{\eta \downarrow 0} \mathbb{Q}\left[\underline{X}_{T} \leq x-\eta\right]=\lim _{\eta \downarrow 0} \mathbb{E}^{\mathbb{Q}}\left[1_{\left\{W_{s} \leq-\eta, \text { for some } 0 \leq s \leq[X]_{T}\right\}}\right]=1,
$$

where the last equality follows from $\mathbb{Q}\left[\underline{W}_{t}<0\right]=1$ for all $t>0$, as shown in (A.1). With $\mathbb{Q} \approx \mathbb{P}$, we conclude that $\mathbb{P}\left[\bar{X}_{T}^{x}>x\right]=\mathbb{Q}\left[\bar{X}_{T}^{x}>x\right]=1$ and $\mathbb{P}\left[\underline{X}_{T}^{x}<x\right]=\mathbb{Q}\left[\underline{X}_{T}^{x}<x\right]=1$.

As a consequence of $\mathbb{P}\left[\bar{X}_{T}^{x}>x\right]=\mathbb{P}\left[\underline{X}_{T}^{x}<x\right]=1$ for all $T>0, \mathbb{P}\left[T_{x}^{x}<T\right]=1$ for all $T>0$. This implies that $T_{x}^{x}<1 / n$ for all $n \in \mathbb{N} \mathbb{P}$-a.s., which yields $T_{x}^{x}=0 \mathbb{P}$-a.s. 


\section{B Optimal Value is Independent of the Finiteness Con- dition}

Most of the results in this paper depend on Xu and Zhou (2013) in which only almost surely finite stopping times are considered. Namely, therein the collection of stopping times is $\overline{\mathcal{T}}=\{\tau \in \mathcal{T}: \mathbb{P}[\tau<\infty]=1\}$. In this paper, it is necessary to consider stopping times that are infinite with positive probability, since some of the explicitly constructed stopping strategies do belong to this category. However, the following result shows that the optimal values are the same with $\mathcal{T}$ and $\overline{\mathcal{T}}$.

Proposition B.1. Let $J$ be defined by 4.9). Then, for any $x>0$,

$$
\sup _{\tau \in \overline{\mathcal{T}}} J(x ; \tau)=\sup _{\tau \in \mathcal{T}} J(x ; \tau) .
$$

Proof. By definition, $\sup _{\tau \in \overline{\mathcal{T}}} J(x ; \tau) \leq \sup _{\tau \in \mathcal{T}} J(x ; \tau)$. For any $\varepsilon>0$, if $\sup _{\tau \in \mathcal{T}} J(x ; \tau)<\infty$ (resp. $\left.\sup _{\tau \in \mathcal{T}} J(x ; \tau)=\infty\right)$, take $\tau^{\prime} \in \mathcal{T}$ such that $J\left(x ; \tau^{\prime}\right)>\sup _{\tau \in \mathcal{T}} J(x ; \tau)-\varepsilon($ resp. $\left.J\left(x ; \tau^{\prime}\right)>1 / \varepsilon\right)$. Consider $\tau_{M}^{\prime}:=\tau^{\prime} \wedge M \in \overline{\mathcal{T}}$ for all $M \in \mathbb{N}$. Then, $u\left(X_{\tau_{M}^{\prime}}^{x}\right)$ converges to $u\left(X_{\tau^{\prime}}^{x}\right)$ a.s., and thus in distribution, as $M \rightarrow \infty$. This in particular implies that for any $y>0, \mathbb{P}\left[u\left(X_{\tau_{M}^{\prime}}^{x}\right)>y\right]$ converges to $\mathbb{P}\left[u\left(X_{\tau^{\prime}}^{x}\right)>y\right]$. By Fatou's lemma,

$$
\begin{aligned}
\liminf _{M \rightarrow \infty} J\left(x ; \tau_{M}^{\prime}\right) & =\liminf _{M \rightarrow \infty} \int_{0}^{\infty} w\left(\mathbb{P}\left[u\left(X_{\tau_{M}^{\prime}}^{x}\right)>y\right]\right) d y \\
& \geq \int_{0}^{\infty} w\left(\lim _{M \rightarrow \infty} \mathbb{P}\left[u\left(X_{\tau_{M}^{\prime}}^{x}\right)>y\right]\right) d y=\int_{0}^{\infty} w\left(\mathbb{P}\left[u\left(X_{\tau^{\prime}}^{x}\right)>y\right]\right) d y=J\left(x ; \tau^{\prime}\right) .
\end{aligned}
$$

It follows that $\sup _{\tau \in \overline{\mathcal{T}}} J(x ; \tau) \geq J\left(x ; \tau^{\prime}\right)$. By the arbitrariness of $\varepsilon>0$, we conclude that $\sup _{\tau \in \overline{\mathcal{T}}} J(x ; \tau)=\sup _{\tau \in \mathcal{T}} J(x ; \tau)$.

It follows from Proposition B.1 that any optimal stopping time constructed in $\mathrm{Xu}$ and Zhou (2013) is automatically an optimal stopping time for $\sup _{\tau \in \mathcal{T}} J(x ; \tau)$.

\section{Proof of Lemma 5.1}

First note that parts of this lemma were derived in $\mathrm{Xu}$ and Zhou (2013), while the cases where $\mathbb{P}\left[\tau_{a b}=\infty\right]>0$ were not dealt with there. This includes " $a=0$ and $b=\infty$ " and " $a=0$ and $b<\infty$ ". For completeness and reader's convenience, we present the proof for all possible cases of $0 \leq a<b \leq \infty$.

Recall that $\tau_{a b}=T_{a}^{x} \wedge T_{b}^{x}$, with $T_{a}^{x}$ and $T_{b}^{x}$ defined as in (4.7), and $u$ is nondecreasing with $u(0)=0$ when $\beta>0$.

(i) Observe that $\tau_{a b}=\infty$ a.s. By (4.6) $), J\left(x ; \tau_{a b}\right)=\int_{0}^{\infty} w(\mathbb{P}[u(0)>y]) d y=0$.

(ii) Thanks to (4.6) $), u\left(X_{\tau_{a b}}^{x}\right)=u(a)$ a.s. It follows that $J\left(x ; \tau_{a b}\right)=\int_{0}^{\infty} w(\mathbb{P}[u(a)>$ $y]) d y=u(a)$.

(iii) We first deal with the case " $a>0$ and $b<\infty$ ". The CDF of $X_{\tau_{a b}}^{x}$ is $F(y)=$ $p^{*} 1_{[a, b)}(y)+1_{[b, \infty)}(y)$ for $y \in[0,1]$, where $p^{*}:=\mathbb{P}\left[X_{\tau_{a b}}^{x}=a\right]=\frac{b-x}{b-a}$ by the optional sampling 
theorem. Note that the use of the optional sampling theorem to find $p^{*}$ requires either $\mathbb{P}\left[T_{a}^{x}<\infty\right]=1$ or $\mathbb{P}\left[T_{b}^{x}<\infty\right]=1$. The former is true here thanks to $a>0$ and (4.6). Then, by (4.10),

$$
J\left(x ; \tau_{a b}\right)=\int_{0}^{a} w(1) u^{\prime}(y) d y+\int_{a}^{b} w\left(1-\frac{b-x}{b-a}\right) u^{\prime}(y) d y+\int_{b}^{\infty} w(0) u^{\prime}(y) d y,
$$

which yields the desired result as $w(0)=0$ and $w(1)=1$.

For the case " $a=0$ and $b<\infty$ ", by the fact that $X^{x}$ does not reach $a=0$ a.s. and (4.6), $X_{\tau_{a b}}^{x}=b 1_{\left\{T_{b}^{x}<\infty\right\}}$ a.s. This, together with $u(0)=0$, gives $u\left(X_{\tau_{a b}}^{x}\right)=u(b) 1_{\left\{T_{b}^{x}<\infty\right\}}$ a.s. It follows that

$$
\begin{aligned}
J\left(x ; \tau_{a b}\right) & =\int_{0}^{\infty} w\left(\mathbb{P}\left[u(b) 1_{\left\{T_{b}^{x}<\infty\right\}}>y\right]\right) d y=\int_{0}^{u(b)} w\left(\mathbb{P}\left[u(b) 1_{\left\{T_{b}^{x}<\infty\right\}}>y\right]\right) d y \\
& =\int_{0}^{u(b)} w\left(\mathbb{P}\left[T_{b}^{x}<\infty\right]\right) d y=w\left(\mathbb{P}\left[T_{b}^{x}<\infty\right]\right) u(b)=w\left(\frac{x}{b}\right) u(b)
\end{aligned}
$$

where the last equality follows from (4.7). Thus, the formula (5.1) still holds for $a=0$.

\section{Proof of Lemma 5.2}

Fix a $D \in \mathcal{B}\left(\mathbb{R}_{+}\right)$. For any $x>0$, define

$$
a(x):=\sup \{a<x: a \in D\}, \quad b(x):=\inf \{b>x: b \in D\} .
$$

If $a(x)=x$ or $b(x)=x$, then $T_{D}^{x}=0$ a.s. and thus $J\left(x ; T_{D}^{x}\right)=u(x)$. If $a(x)<x<b(x)$, then $T_{D}^{x}=\inf \left\{t \geq 0: X_{t}^{x} \notin(a(x), b(x))\right\}$. We then deduce from Lemma 5.1 that

$$
J\left(x ; T_{D}^{x}\right)=\left\{\begin{array}{lr}
u(x), & \text { if } a(x)=x \text { or } b(x)=x, \\
u(a(x))+w\left(\frac{x-a(x)}{b(x)-a(x)}\right)(u(b(x))-u(a(x))), & \text { if } a(x)<x<b(x)<\infty, \\
u(a(x)), & \text { if } 0<a(x)<x<b(x)=\infty, \\
0, & \text { if } 0=a(x)<x<b(x)=\infty .
\end{array}\right.
$$

Now, note that with $D_{\mathbb{Q}}:=\{q \in \mathbb{Q}: a \leq q \leq b$ for some $a, b \in D\}$,

$a(x)=\sup \left\{q<x: q \in D_{\mathbb{Q}}\right\}=\sup _{q \in D_{\mathbb{Q}}} q 1_{(q, \infty)}(x), \quad b(x)=\inf \left\{q>x: q \in D_{\mathbb{Q}}\right\}=\inf _{q \in D_{\mathbb{Q}}} q 1_{(0, q)}(x)$.

It follows that $x \mapsto a(x)$ and $b \mapsto b(x)$ are both Borel measurable. We then conclude form (D.2) that $x \mapsto J\left(x ; T_{D}^{x}\right)$ is Borel measurable, i.e. Assumption 2.1 (i) is satisfied.

Fix a sequence $\left\{D_{n}\right\}_{n \in \mathbb{N}}$ in $\mathcal{B}\left(\mathbb{R}_{+}\right)$such that $D_{n} \subseteq D_{n+1}$ for all $n \in \mathbb{N}$. For any $x>0$, consider $a(x)$ and $b(x)$ as in (D.1) with $D:=\bigcup_{n \in \mathbb{N}} D_{n}$. Moreover, we define

$$
a_{n}(x):=\sup \left\{a<x: a \in D_{n}\right\}, \quad b_{n}(x):=\inf \left\{b>x: b \in D_{n}\right\}, \quad \text { for all } n \in \mathbb{N} .
$$


Since $D_{n} \subseteq D_{n+1}$ for all $n \in \mathbb{N}$, we have $a_{n}(x) \uparrow a(x)$ and $b_{n}(x) \downarrow b(x)$ as $n \rightarrow \infty$. For each $n \in \mathbb{N}$, by the same argument above (D.2),

$$
J\left(x ; T_{D_{n}}^{x}\right)=\left\{\begin{array}{lr}
u(x), & \text { if } a_{n}(x)=x \text { or } b_{n}(x)=x, \\
u\left(a_{n}(x)\right)+w\left(\frac{x-a_{n}(x)}{b_{n}(x)-a_{n}(x)}\right)\left(u\left(b_{n}(x)\right)-u\left(a_{n}(x)\right)\right), & \text { if } a_{n}(x)<x<b_{n}(x)<\infty \\
u\left(a_{n}(x)\right), & \text { if } 0<a_{n}(x)<x<b_{n}(x)=\infty \\
0, & \text { if } 0=a_{n}(x)<x<b_{n}(x)=\infty .
\end{array}\right.
$$

From the above formula and ( $\overline{\mathrm{D} .2}$ ), one may deduce from the continuity of $u$ and $w$ and the convergence $a_{n}(x) \uparrow a(x)$ and $b_{n}(x) \downarrow b(x)$ that

$$
\lim _{n \rightarrow \infty} J\left(x ; T_{D_{n}}^{x}\right)=J\left(x ; T_{D}^{x}\right) .
$$

Indeed, the only nontrivial case is " $a(x)=b(x)=x$ while $a_{n}(x)<x<b_{n}(x)$ for all $n \in \mathbb{N}$ ". In this case,

$$
\begin{aligned}
\lim _{n \rightarrow \infty} J\left(x ; T_{D_{n}}^{x}\right) & =\lim _{n \rightarrow \infty}\left[u\left(a_{n}(x)\right)+w\left(\frac{x-a_{n}(x)}{b_{n}(x)-a_{n}(x)}\right)\left(u\left(b_{n}(x)\right)-u\left(a_{n}(x)\right)\right)\right] \\
& =u(x)+\lim _{n \rightarrow \infty}\left[w\left(\frac{x-a_{n}(x)}{b_{n}(x)-a_{n}(x)}\right)\left(u\left(b_{n}(x)\right)-u\left(a_{n}(x)\right)\right)\right]=u(x)=J\left(x ; T_{D}^{x}\right),
\end{aligned}
$$

where the third equality follows from $a(x)=b(x)=x$ and $w$ being a bounded function. Then (D.3) in particular implies that Assumption 2.1 (ii) is satisfied.

\section{References}

Birnbaum, M. H. and McIntosh, W. R. (1996), 'Violations of branch independence in choices between gambles', Organizational Behavior and Human Decision Processes 67(1), 91-110.

Björk, T., Khapko, M. and Murgoci, A. (2017), 'On time-inconsistent stochastic control in continuous time', Finance and Stochastics 21(2), 331-360.

Camerer, C. F. and Ho, T. (1994), 'Violations of the betweenness axiom and nonlinearity in probability', Journal of Risk and Uncertainty 8(2), 167-96.

Ebert, S. and Strack, P. (2015), 'Until the bitter end: On prospect theory in a dynamic context', American Economic Review 105(4), 1618-33.

Ebert, S. and Strack, P. (2017), 'Never, ever getting started: on prospect theory without commitment'. Available at https://ssrn.com/abstract $=2765550$.

Ebert, S., Wei, W. and Zhou, X. Y. (2017), 'Discounting, diversity, and investment'. Available at https://ssrn.com/abstract $=2840240$.

Ekeland, I. and Lazrak, A. (2006), 'Being serious about non-commitment: subgame perfect equilibrium in continuous time'. Available at http://arxiv.org/abs/math/0604264. 
He, X. D. and Zhou, X. Y. (2016), 'Hope, fear, and aspirations', Math. Finance 26(1), 3-50.

He, X., Hu, S., Obłój, J. and Zhou, X. Y. (2017), 'Path-dependent and randomized strategies in barberis' casino gambling model', Operations Research 65, 97-103.

Hu, Y., Jin, H. and Zhou, X. Y. (2012), 'Time-inconsistent stochastic linear-quadratic control', SIAM Journal on Control and Optimization 50, 1548-1572.

Hu, Y., Jin, H. and Zhou, X. Y. (2017), 'Time-inconsistent stochastic linear-quadratic control: Characterization and uniqueness of equilibrium', SIAM Journal on Control and Optimization 55, 1261-1579.

Huang, Y.-J. and Nguyen-Huu, A. (2016), Time-consistent stopping under decreasing impatience, Technical report, Dublin City University and École Polytechnique. Available at http://arxiv.org/abs/1502.03998.

Karatzas, I. and Shreve, S. E. (1991), Brownian motion and stochastic calculus, Vol. 113 of Graduate Texts in Mathematics, second edn, Springer-Verlag, New York.

Karatzas, I. and Shreve, S. E. (1998), Methods of mathematical finance, Vol. 39 of Applications of Mathematics (New York), Springer-Verlag, New York.

Karnam, C., Ma, J. and Zhang, J. (2016), 'Dynamic approaches for some time inconsistent problems'. Available at https://arxiv.org/abs/1604.03913.

Lattimore, P. K., Baker, J. R. and Witte, A. D. (1992), 'The influence of probability on risky choice: A parametric examination', Journal of Economic Behavior and Organization 17(3), 377-400.

Prelec, D. (1998), 'The probability weighting function', Econometrica 66(3), 497-528.

Shiryaev, A. (1978), Optimal stopping rules, Springer-Verlag, New York.

Shiryaev, A., Xu, Z. and Zhou, X. Y. (2008), 'Thou shalt buy and hold', Quant. Finance 8(8), $765-776$.

Strotz, R. H. (1955-56), 'Myopia and inconsistency in dynamic utility maximization', The Review of Economic Studies 23, 165-180.

Tversky, A. and Kahneman, D. (1992), 'Advances in prospect theory: Cumulative representation of uncertainty', Journal of Risk and Uncertainty 5, 297-323.

Wu, G. and Gonzalez, R. (1996), 'Curvature of the probability weighting function', Management Science 42(12), 1676-1690.

Xu, Z. Q. and Zhou, X. Y. (2013), 'Optimal stopping under probability distortion', Ann. Appl. Probab. 23(1), 251-282.

Yong, J. (2012), 'Time-inconsistent optimal control problems and the equilibrium HJB equation', arXiv preprint arXiv:1204.0568 . 